\title{
TRANSFERÊNCIA DE ELÉTRONS EM SISTEMAS INORGÂNICOS DE VALÊNCIA MISTA
}

\author{
Reginaldo C. Rocha e Henrique E. Toma* \\ Instituto de Química, Universidade de São Paulo, CP 26077, 05513-970, São Paulo - SP
}

Recebido em 3/5/01; aceito em 6/7/01

ELECTRON TRANSFER IN INORGANIC MIXED-VALENCE SYTEMS. This article reports on some basic and conceptual principles concerning electron transfer (ET) and/or intervalence transfer (IT) phenomena in inorganic mixed-valence systems.

Keywords: electron transfer; intervalence charge transfer; mixed-valence.

\section{INTRODUÇÃO}

Em um contexto bastante abrangente, os materiais de valência mista $^{1}$ são aqueles que contêm íons em mais de um estado de oxidação formal em uma mesma unidade molecular. Existem muitos exemplos na bioquímica (p. ex. metaloenzimas contendo múltiplos sítios de transferência eletrônica e clusters de ferro-enxofre), na química mineral (p. ex. magnetita), ou envolvendo sólidos moleculares (p. ex. cerâmicas condutoras).

De fato, a existência de sistemas de valência mista remonta à história evolucionária da Terra e de outros planetas. Além disso, uma variedade de minerais de valência mista tem atraído atenção desde a antiguidade. O caráter de valência mista é, na verdade, responsável pela coloração de vários minerais bem conhecidos. Por exemplo, o controle dos estados de oxidação do ferro em seus óxidos $\left(\mathrm{FeO}, \mathrm{Fe}_{3} \mathrm{O}_{4}\right.$, $\mathrm{Fe}_{2} \mathrm{O}_{3}$ ) foi elegantemente utilizado na pintura de vasos pelos gregos antigos para se produzir o preto e o vermelho característicos das cerâmicas áticas ${ }^{2}$.

No entanto, somente há pouco mais de três décadas é que os primeiros artigos de revisão acerca dos compostos de valência mista apareceram na literatura ${ }^{3-5}$, na tentativa de se tratar os materiais de valência mista como uma classe separada de compostos, cujas propriedades podiam ser correlacionadas com as estruturas moleculares e eletrônicas de seus componentes. Desde então, o fenômeno de valência mista tem atraído o interesse de uma ampla variedade de cientistas, que se estende de químicos a físicos, de biólogos a geólogos.

A química inorgânica, em especial, tem colaborado com grande parte do conhecimento teórico e experimental acumulado neste campo, principalmente através do estudo dos complexos polinucleares de valência mista ${ }^{6-9}$. Os compostos de coordenação tiveram, de fato, uma história antiga dentro deste assunto. Por exemplo, o azul da Prússia, $\mathrm{Fe}\left[\mathrm{Fe}(\mathrm{CN})_{6}\right]^{-}$, que é um complexo de valência mista com uma estrutura do tipo $\mathrm{Fe}(\mathrm{II})-\mathrm{Fe}$ (III) mediada por pontes de cianeto, foi um dos primeiros materiais químicos descritos na literatura há quase três séculos ${ }^{10}$. Na realidade, o azul da Prússia é protótipo de uma extensa série de compostos do tipo $\mathrm{M}_{x}{ }_{x}\left[\mathrm{M} \text { ' }(\mathrm{CN})_{5}(\mathrm{~L})\right]_{y}$, onde M' e M" são íons metálicos em estados de oxidação distintos, e L, um ligante qualquer ${ }^{11,12}$. Esses compostos têm despertado interesse pela capacidade de formar filmes ${ }^{12,13}$ finos e condutores, apresentando variações cromáticas em função das mudanças nos estados de oxidação ${ }^{12}$.

*e-mail: henetoma@iq.usp.br
Todavia, somente no início dos anos 70 do século passado é que foram reportados os primeiros complexos planejados e preparados em laboratório, bem como estudados por meio de técnicas modernas de espectroscopia e eletroquímica. Esse é o caso do dímero $\mu$-pirazinabis $\{$ pentaaminrutênio $\}(5+),\left[\left(\mathrm{NH}_{3}\right)_{5} \mathrm{Ru}(\mathrm{pz}) \mathrm{Ru}\left(\mathrm{NH}_{3}\right)_{5}\right]^{5+}$, que ficou conhecido como íon de Creutz-Taube ${ }^{14}$ (Esquema 1), e do monocátion biferroceno contendo grupos $\eta^{5}$-ciclopentadienil como ponte ${ }^{15}$, $[(\mathrm{cp}) \mathrm{Fe}(\mathrm{cp}-\mathrm{cp}) \mathrm{Fe}(\mathrm{cp})]^{+}$(Esquema 2).

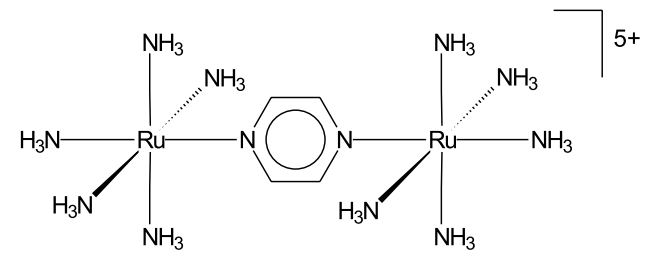

Esquema 1. O íon complexo de Creutz-Taube

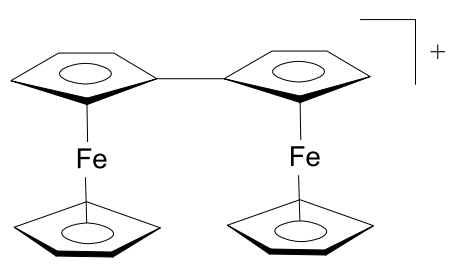

Esquema 2. O cátion biferroceno

Desde então, químicos inorgânicos têm demonstrado grande interesse na exploração de moléculas em que dois ou mais centros metálicos conectados por meio de um ligante-ponte (que normalmente é um orgânico heterocíclico, mas pode variar desde entidades mono- ou diatômicas até ligantes polifuncionais) apresentem alguma comunicação eletrônica metal-metal do tipo doador-aceitador.

O grande sucesso dos sistemas de valência mista, em particular daqueles baseados em oligômeros de metais de transição, deve-se ao importante papel destas espécies como modelos ideais na investigação de processos de transferência eletrônica inter- e/ou intramoleculares. Isso porque a complexidade cinética e dinâmica e o desconhecimento da configuração nuclear/geométrica em reações de transferência de elétrons intermoleculares tornam impraticável o estudo direto de tais sistemas. Por outro lado, o emprego apropriado de complexos de valência mista tem sido eficaz dentro desse propósito, uma vez que a 
natureza química do ligante-ponte mediador pode ser convenientemente escolhida e a estrutura molecular dessas espécies é conhecida na etapa de transferência eletrônica. Ademais, a estrutura eletrônica dos membros que os compõem pode ser modulada por meio de perturbações químicas de esfera interna ou de esfera externa.

Essa abordagem tem sido aplicada adequadamente para espécies metal-orgânicas envolvendo principalmente alguns dos elementos da primeira e da segunda séries de transição (na maioria, complexos homo- e heteronucleares contendo amina ${ }^{6-9,16-19}$, carboxilato ${ }^{6,9,16,18,20-}$ ${ }^{23}$, cianeto $^{6,7,9,16,17,19,24}$, fosfina ${ }^{25}$ e polipiridil-derivados ${ }^{6,8,9,26-30}$ como coligantes e uma grande variedade de ligantes-ponte envolvendo grupos carboxilato, ciano, halo, hidroxo, nitro, oxo, e, principalmente, heterocíclicos orgânicos nitrogenados ${ }^{6-9}$; ligantes-ponte oxigenados não-inocentes, sejam eles do tipo dioxolenos ${ }^{8,31,32}$ ou polifuncionais $^{33,34}$, e sulfurados s,8,17,35,36 $^{6}$ também têm sido empregados). Complexos dos elementos de transição do Grupo 8 (em particular,

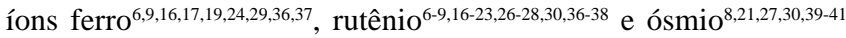
com estados de oxidação +II e +III - configuração eletrônica $d^{6} / d^{5}$ de spin baixo) são especialmente interessantes devido a algumas características tais como: cinética favorável, estabilidade termodinâmica, viabilidade sintética, e química redox bastante flexível ${ }^{6,38,42,43}$. Além disso, no estado de oxidação formal +II, tais íons são fortes doadores- $\pi$ que interagem eficientemente com ligantes aceitadores- $\pi^{6,38,43}$.

As características combinadas dos complexos inorgânicos de valência mista lhes atribuem propriedades supramoleculares originais com aplicabilidade em diversas áreas: conversão de energia, novos materiais, catálise e eletrônica molecular, entre outros ${ }^{44}$.

\section{TRANSFERÊNCIA DE ELÉTRONS}

As reações envolvendo transferência de elétrons são onipresentes em sistemas biológicos, físicos e químicos. Na natureza, a ocorrência dessas reações está intimamente relacionada aos processos de transferência de energia, a exemplo do que se dá no centro de reação fotossintética, na fosforilação oxidativa, e em muitos outros eventos com múltiplas etapas de transferência de elétrons acopladas, tal como ocorre na cadeia respiratória.

Em última análise, o fenômeno de transferência eletrônica se manifesta nos principais processos responsáveis pela manutenção da vida. A compreensão das interações desse tipo é, portanto, crucial no entendimento de mecanismos de reações fundamentais, o que explica porque esta constitui uma das áreas de pesquisa mais amplas e ativas no âmbito das ciências moleculares modernas ${ }^{45}$.

As reações de transferência de elétrons podem variar desde muito simples até bastante complexas. No caso mais simples (como aquele representado na Eq. 1 ${ }^{46}$, um elétron é transferido em uma reação de troca (self-exchange) entre dois íons possuindo a mesma constituição molecular mas diferentes estados de oxidação. Nesse caso, não existe mudança química no sistema.

$$
\begin{gathered}
{\left[\mathrm{Ru}^{\mathrm{III}}(\text { bpy })_{3}\right]^{3+}+\left[\mathrm{Ru}^{\mathrm{II}}(\mathrm{bpy})_{3}\right]^{2+}} \\
\int_{\left[\mathrm{Ru}^{\mathrm{II}}(\mathrm{bpy})_{3}\right]^{2+}+\left[\mathrm{Ru}^{\mathrm{III}}(\mathrm{bpy})_{3}\right]^{3+}}\left(k=1,2 \times 10^{9} \mathrm{~mol}^{-1} \mathrm{dm}^{3} \mathrm{~s}^{-1}\right)
\end{gathered}
$$

Em contraste, a Eq. 2 ilustra um exemplo onde a reação total é bastante complexa e envolve várias etapas com transferência de elétrons, bem como outras etapas de reações intermediárias ${ }^{47}$.

$$
\begin{gathered}
\mathrm{FeCl}_{3}+7 \mathrm{PhLi} \\
\downarrow
\end{gathered}
$$

$\left[\mathrm{FePh}_{4}\right]^{4}\left(\mathrm{Li}^{+}\right)_{4}+3 / 2 \mathrm{Ph}-\mathrm{Ph}+3 \mathrm{LiCl}$
No começo dos anos 50, Taube ${ }^{48,49}$ propôs uma distinção essencial entre duas classes de reações de transferência de elétrons: as que ocorrem segundo um mecanismo de esfera externa, sem quebra ou formação intermediária de ligação química; e aquelas que se processam de acordo com um mecanismo de esfera interna, onde um ligante do tipo ponte conecta os grupos doador e aceitador em um complexo binuclear intermediário durante a transferência eletrônica.

Exemplos clássicos de reações de transferência de elétrons de esfera externa ${ }^{50}$ são dados pelas Eqs. $1^{46}$ e $3{ }^{51}$.

$$
\begin{gathered}
{\left[\mathrm{Fe}^{\mathrm{II}}(\mathrm{CN})_{6}\right]^{4-}+\left[\mathrm{Ir}^{\mathrm{IV}} \mathrm{Cl}_{6}\right]^{2-}} \\
\prod_{\left(k=4,1 \times 10^{5} \mathrm{~mol}^{-1} \mathrm{dm}^{3} \mathrm{~s}^{-1}\right)} \\
{\left[\mathrm{Fe}^{\mathrm{III}}(\mathrm{CN})_{6}\right]^{3-}+\left[\mathrm{Ir}^{\mathrm{III}} \mathrm{Cl}_{6}\right]^{3-}}
\end{gathered}
$$

Um exemplo bem conhecido de reação de esfera interna é aquele que se dá entre os íons complexos $\left[\mathrm{Cr}^{\mathrm{II}}\left(\mathrm{H}_{2} \mathrm{O}\right)_{6}\right]^{2+} \mathrm{e}\left[\mathrm{Co}{ }^{\mathrm{III}} \mathrm{Cl}\left(\mathrm{NH}_{3}\right)_{5}\right]^{2+}{ }^{49}$ $\mathrm{O}$ íon $\mathrm{Cr}^{\mathrm{II}}$, sendo bastante lábil, troca rapidamente um ligante água pelo ligante cloreto do íon inerte $\mathrm{Co}^{\mathrm{III}}$ (Eq. 4). Após a etapa de transferência eletrônica no intermediário binuclear contendo o cloreto como ponte (Eq. 5), a labilidade é invertida. O íon $\mathrm{Co}^{\mathrm{II}}$ é lábil e, portanto, perde o ligante cloreto, que permanece coordenado ao íon inerte $\mathrm{Cr}^{\text {III }}$ (Eq. 6) ${ }^{49}$.

$$
\begin{aligned}
& {\left[\mathrm{Cr}^{\mathrm{II}}\left(\mathrm{H}_{2} \mathrm{O}\right)_{6}\right]^{2+}+\left[\mathrm{Co}{ }^{\mathrm{III}} \mathrm{Cl}\left(\mathrm{NH}_{3}\right)_{5}\right]^{2+}} \\
& \downarrow \\
& {\left[\left(\mathrm{H}_{2} \mathrm{O}\right)_{5} \mathrm{Cr}^{\mathrm{II}} \leftarrow \mathrm{Cl}-\mathrm{Co}^{\mathrm{III}}\left(\mathrm{NH}_{3}\right)_{5}\right]^{4+}} \\
& \sqrt{\|} \\
& {\left[\left(\mathrm{H}_{2} \mathrm{O}\right)_{5} \mathrm{Cr} \text { III }-\mathrm{Cl} \rightarrow \mathrm{Co}^{\mathrm{II}}\left(\mathrm{NH}_{3}\right)_{5}\right]^{4+}} \\
& \downarrow \\
& {\left[\mathrm{Cr}{ }^{\mathrm{III}} \mathrm{Cl}\left(\mathrm{H}_{2} \mathrm{O}\right)_{5}\right]^{2+}+\left[\mathrm{Co}^{\mathrm{II}}\left(\mathrm{H}_{2} \mathrm{O}\right)\left(\mathrm{NH}_{3}\right)_{5}\right]^{2+}} \\
& \downarrow^{\mathrm{H}+, \mathrm{H} 2 \mathrm{O}} \\
& 5 \mathrm{NH}_{4}^{+}+\left[\mathrm{Co}^{\mathrm{II}}\left(\mathrm{H}_{2} \mathrm{O}\right)_{6}\right]^{2+}
\end{aligned}
$$

Entretanto, os modelos mais simples para reações de transferência de elétrons de esfera interna são os complexos de valência mista, cujo exemplo pioneiro é representado pelo íon de Creutz-Taube ${ }^{14}$ (Esquema 1).

Em um complexo desse tipo, a assimetria redox introduz uma perturbação eletrônica no sistema molecular, a partir da qual a oscilação contínua de cargas é promovida. A Eq. 8 ilustra o processo para o análogo do íon de Creutz-Taube contendo 4,4'-bipiridina como ligante-ponte ${ }^{52-55}$.
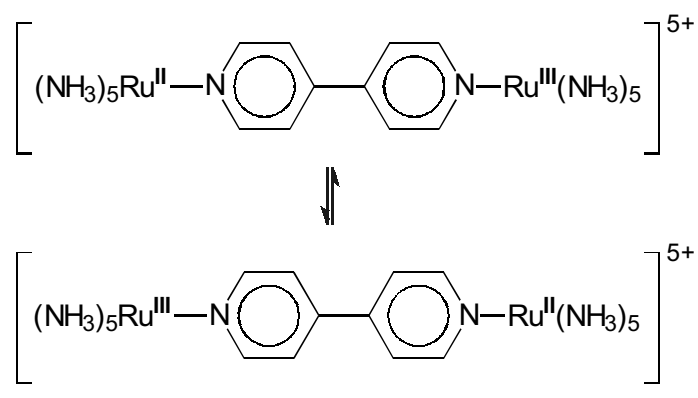

A teoria cinética de Marcus ${ }^{56-58}$ para reações de transferência de elétrons de esfera externa e a teoria perturbacional de $\mathrm{Hush}^{5,59,60}$ para complexos de valência mista do tipo doador-aceitador são ferramentas extremamente úteis na compreensão do comportamento de tais sistemas ${ }^{61}$. 
Um aspecto da maior importância nesse tipo de reação é a relação entre a velocidade de transferência de elétrons ${ }^{62}$ e a exergonicidade, $\Delta G^{0}{ }^{63}$ Quando uma reação é bastante exergônica $\left(\Delta G^{0} \ll<\right)$, pode-se assegurar que a transferência de elétrons ocorrerá com uma alta constante de velocidade; em uma reação endergônica $\left(\Delta G^{0}>>0\right)$, pode-se eliminar a possibilidade de transferência eletrônica. Porém, a situação entre esses dois extremos é quase sempre de muito interesse. Nota-se ainda que reações moderadamente endergônicas podem ocorrer se elas forem dirigidas por métodos externos (p. ex. óptica ou eletroquimicamente). Nos casos isoergônicos (onde $\Delta G^{0}=0$ ), tais como nas Eqs. 1 e 8 , a barreira cinética $\left(\Delta G^{\ddagger}\right)$ não é zero e sua determinação experimental é bastante relevante dentro dos modelos de Marcus e Hush ${ }^{5,57-60}$.

Reações de transferência de elétrons de esfera interna são mais difíceis de se analisar do ponto de vista quântico, mas seus mecanis-
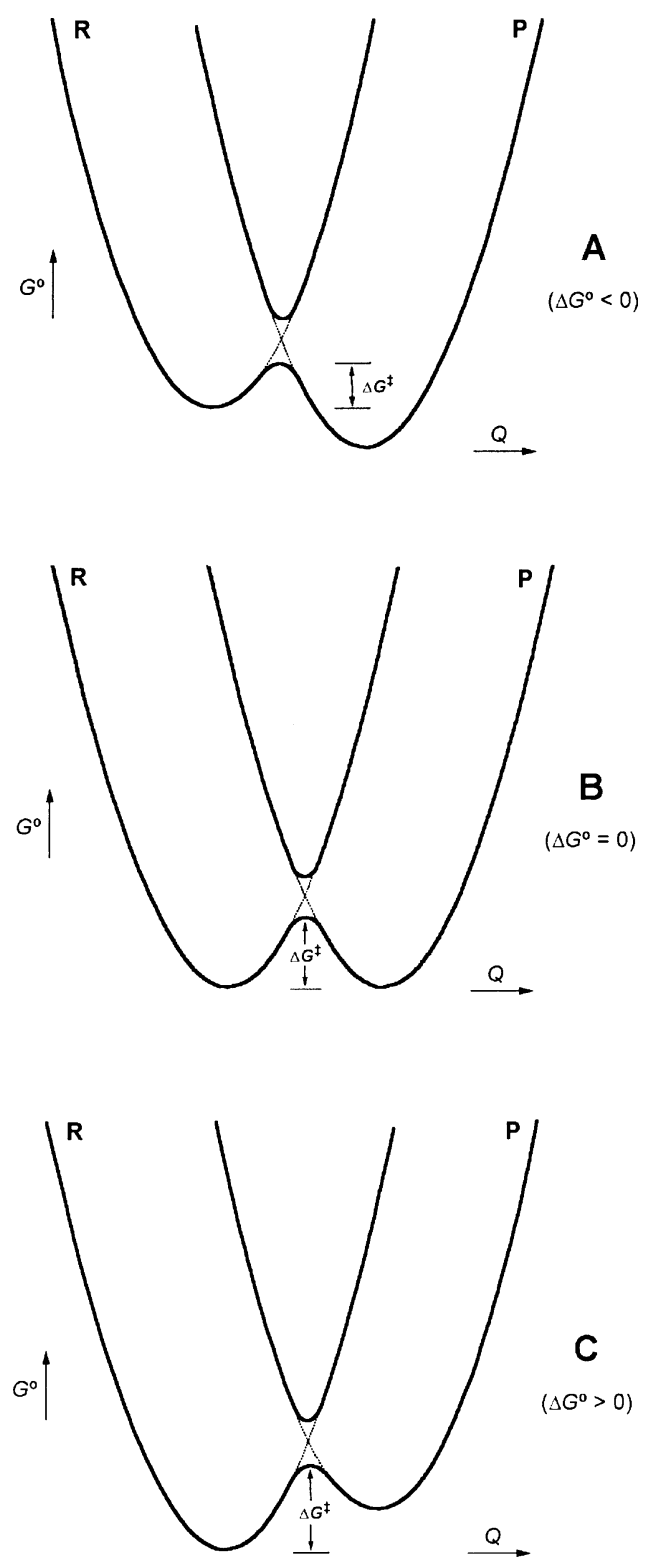

Figura 1. Diagramas ${ }^{75}$ ilustrativos de energia livre $\left(\mathrm{G}^{\circ}\right)$ em função de coordenadas de reação (Q) para três tipos de sistemas: (A) assimétrico (doador $\neq$ aceitador) e exergônico $\left(\Delta \mathrm{G}^{o}<0\right)$; $(\boldsymbol{B})$ simétrico (doador $=$ aceitador) e isoergônico $\left(\Delta \mathrm{G}^{o}=0\right) ;$ e $(\boldsymbol{C})$ assimétrico (doador $\neq$ aceitador) e endergônico $\left(\Delta \mathrm{G}^{o}>0\right)$. Nos diagramas, $\Delta \mathrm{G}^{\ddagger}$ é a energia livre de ativação da reação mos moleculares complexos têm sido monitorados em muitos casos. A compreensão desses mecanismos tem sido aprofundada por meio de estudos da química dos complexos metálicos inorgânicos, particularmente aqueles realizados por Taube ${ }^{43,49,65,66}$, Sutin $^{67-69}$, Haim ${ }^{16,70}$, e colaboradores.

As reações de transferência de elétrons fotoinduzidas ${ }^{26,30,71-74}$, por outro lado, podem ser altamente endergônicas, o que permite estudar processos que seriam termodinamicamente proibidos, bem como a cinética a eles associada.

O estudo da transferência eletrônica biológica a longas distâncias tem sido especialmente desafiador nas últimas décadas, tanto do ponto de vista teórico ${ }^{58,77-79}$ como do experimental ${ }^{34,80,81}$. Tunelamento eletrônico e reconhecimento molecular são alguns dos conceitos essenciais que têm sido estabelecidos por meio de técnicas experimentais poderosas e precisas, tais como medidas de velocidade de reações de transferência de elétrons dependente da temperatura e engenharia de proteínas ${ }^{34,80,81}$ e de fragmentos de DNA $^{82,83}$ modificados por incorporação de complexos inorgânicos.

Atualmente, além de vários livros ${ }^{66,71,73,78,84,85}$ e enciclopédias ${ }^{86}$ enfatizando aspectos inorgânicos, orgânicos, físicos e teóricos, um grande número de artigos de revisão sobre vários aspectos de reações de transferência de elétrons encontram-se disponíveis (v. ref. 9 e referências citadas).

\section{Transferência de Intervalência}

A transferência de intervalência é um caso particular de transferência de elétrons intramolecular que se manifesta em sistemas moleculares contendo centros redox em diferentes estados de oxidação ${ }^{9}$. Por isso é que, quando um elétron é transferido de um sítio doador (p. ex. menor estado de oxidação; redutor) para outro aceitador (p. ex. maior estado de oxidação; oxidante), diz-se que houve uma transição com mudança "entre valências", daí o termo intervalência. Interações dessa natureza também são chamadas de transferência de carga de intervalência (IVCT) ou transferência de carga metal-metal (MMCT). Em química inorgânica, esse fenômeno tem sido estudado principalmente em complexos bi- ou polinucleares de valência mista $^{6-9}$, o que constitui o tema central deste artigo.

Uma transição de intervalência é um processo induzido por luz que, normalmente, pode ser monitorado através de uma banda de absorção de baixa energia, na região do infravermelho próximo. Essas bandas comumente apresentam uma estrutura vibracional bastante complexa, com dependência de esfera interna e do solvente.

Deve ser mencionado que bandas de intervalência também podem ser observadas em complexos de esfera externa, do tipo $\left[\mathrm{Ru}^{\mathrm{III}}\left(\mathrm{NH}_{3}\right)_{6}\right]\left[\mathrm{Fe}^{\mathrm{II}}(\mathrm{CN})_{5}(\mathrm{~L})\right]$, onde a excitação óptica entre os centros metálicos se processa sem a intermediação de ligantes-ponte ${ }^{87}$.

A partir da análise dos processos de intervalência (principalmente através do formalismo perturbacional clássico de Mulliken-Hush ${ }^{5,59,60}$, embora modelos de acoplamento vibrônico mais recentes tenham sido empregados ${ }^{88,89,90}$ ), químicos e físicos têm aprendido muito acerca da natureza dos fenômenos de transferência de elétrons óptica (ou fotoinduzida) e dos mecanismos de acoplamento eletrônico e deslocalização em sistemas do tipo doador-aceitador. Isso é particularmente verdadeiro para sistemas de interação fraca (cargas localizadas) e de acoplamento forte (cargas deslocalizadas).

\section{SISTEMAS DE VALÊNCIA MISTA}

\section{Tipos e Classificação de Sistemas de Valência Mista}

Nas seções anteriores foi descrito que, para uma reação de transferência de elétrons ser exergônica $\left(\Delta G^{\text {o }}<0\right.$; termodinamicamente 
favorável) ou endergônica ( $\Delta G^{\mathrm{o}}>0$; termodinamicamente desfavorável), o sistema deve ser assimétrico. Isto é, as espécies doadora e aceitadora de elétrons que o compõem devem ser química e/ou eletronicamente distintas. Isso porque, em ambos os casos, os estados eletrônicos dos reagentes e dos produtos devem ser diferentes para que ocorra variação de energia durante o processo de transferência eletrônica.

No caso específico de um complexo binuclear assimétrico de valência mista, este pode conter dois íons metálicos de elementos diferentes (sistema assimétrico heteronuclear) ou dois íons metálicos do mesmo elemento em diferentes ambientes químicos (sistema assimétrico homonuclear). Exemplos desses sistemas são dados pelas Eqs. 9 e 10, respectivamente.

$\left[\mathrm{Cl}(\mathrm{bpy})_{2} \mathrm{Os}^{\mathrm{II}}-\mathrm{L}-\mathrm{Ru}^{\mathrm{III}}(\mathrm{bpy})_{2} \mathrm{Cl}\right]^{n}$
$\left[\mathrm{Cl}(\mathrm{bpy})_{2} \mathrm{Os}^{\mathrm{III}}-\mathrm{L}-\mathrm{Ru}^{\mathrm{II}}(\mathrm{bpy})_{2} \mathrm{Cl}\right]^{n}$
$\left[\left(\mathrm{NH}_{3}\right)_{5} \mathrm{Ru}^{\mathrm{II}}-\mathrm{L}-\mathrm{Ru}^{\mathrm{III}} \mathrm{Cl}(\mathrm{bpy})_{2}\right]^{n+1}$
$\left[\left(\mathrm{NH}_{3}\right)_{5} \mathrm{Ru}^{\mathrm{III}}-\mathrm{L}-\mathrm{Ru}^{\mathrm{II}} \mathrm{Cl}(\mathrm{bpy})_{2}\right]^{n+1}$

(onde $n=3$ se L for neutro)

Uma reação isoergônica, por sua vez, quase sempre envolve um sistema simétrico homonuclear; isto é, um sistema molecular contendo duas unidades terminais idênticas, a exemplo do íon de CreutzTaube (Esquema 1) e do dímero do tipo ( $\mu$-L)bis $\left\{\right.$ bis $\left(2,2^{\prime}\right.$ bipiridina)clororutênio\}(II,III), conforme representado na Eq. 11.

$\left[\mathrm{Cl}(\mathrm{bpy})_{2} \mathrm{Ru}^{\mathrm{II}}-\mathrm{L}-\mathrm{Ru}^{\mathrm{III}}(\mathrm{bpy})_{2} \mathrm{Cl}\right]^{n}$

$\sqrt{\|}$

$\left[\mathrm{Cl}(\mathrm{bpy})_{2} \mathrm{Ru}^{\mathrm{III}}-\mathrm{L}-\mathrm{Ru}^{\mathrm{II}}(\mathrm{bpy})_{2} \mathrm{Cl}\right]^{n}$

(onde $n=3$ se L for neutro)

Os complexos de valência mista são classificados de acordo com a intensidade da interação eletrônica entre os centros metálicos doador e aceitador. Robin e Day ${ }^{3}$ propuseram três categorias de sistemas de valência mista:

Classe I. A interação entre os centros redox é tão fraca que o sistema de valência mista exibe somente as propriedades individuais de cada um de seus componentes redox isolados (similar à soma das unidades monômeras de partida). Essa classe é representada principalmente por sistemas contendo moléculas de ponte isolantes (p. ex. cadeias saturadas) e/ou longas distâncias entre os sítios doador e aceitador (p. ex. proteínas quimicamente modificadas pela coordenação de complexos de metais de transição em resíduos periféricos, onde $d_{\mathrm{ET}}$ é tipicamente maior que 12 $15 \AA)^{34,77-81}$. Nesses casos, a reação é não-adiabática $(\kappa<$ $1)^{92}$ e o mecanismo de transferência de elétrons se dá essencialmente por tunelamento eletrônico ${ }^{78,93,94}$ (Figura 2A);

Classe II. Há um acoplamento eletrônico fraco, de tal forma a perturbar as características originais dos centros redox. O sistema de valência mista normalmente passa a apresentar novas propriedades não associadas às espécies de par- tida isoladamente. Essa classe corresponde à maioria das moléculas de interesse no contexto da química de valência mista ${ }^{6-9}$. Nela, a magnitude da comunicação entre os centros doador e aceitador é grande o suficiente para tornar o sistema adiabático (com $\kappa=1)^{92}$ mas as cargas permanecem essencialmente localizadas (Figura 2B). A teoria de Marcus-Hush se aplica a sistemas desta classe;

Classe III. A interação eletrônica entre os centros é bastante forte e o sistema de valência média passa a exibir novas propriedades, que são totalmente diferentes daquelas de suas unidades componentes. Diferentemente dos sistemas de classe II, onde as características particulares dos monômeros isolados são apenas ligeiramente modificadas, aqui elas desaparecem por completo. Os sistemas que se incluem nessa classe são totalmente adiabáticos $(\kappa=$ $1)^{92}$ e apresentam deslocalização total de cargas (p. ex. complexos do tipo $\left.\mathrm{M}^{\mathrm{II} / 2}-\mathrm{L}-\mathrm{M}^{\mathrm{II} / 2}\right)^{6,7,9}$. Como conseqüência, os estados eletrônicos simétricos definidos pelas espécies reagente e produto deixam de existir para dar lugar a uma nova entidade onde a transferência de elétrons se manifesta, na verdade, como uma transferência de carga interna do tipo $\pi \rightarrow \pi^{*}$ (Figura 2C). Exemplos típicos de sistemas eletronicamente deslocalizados são fornecidos pelos complexos da série $\left[\mathrm{Ru}_{3} \mathrm{O}\left(\mathrm{CH}_{3} \mathrm{CO}_{2}\right)_{6}(\mathrm{~L})_{3}\right]^{x}$, onde a interação metal-metal é suficientemente forte para justificar a denominação de cluster metálico ${ }^{23}$. A teoria de Marcus-Hush não se aplica a sistemas desta classe.

Os critérios experimentais para se distinguir particularmente sistemas de classe II e classe III serão explorados mais adiante, conjuntamente com a análise dos fatores de esferas interna e externa que influenciam a energia da transição de intervalência segundo o modelo de Hush.

\section{Reação de Comproporcionamento}

A manifestação mais direta da comunicação eletrônica em complexos de valência mista é a separação de potenciais redox para os centros metálicos em ambientes químicos aparentemente idênti$\cos ^{6-9}$.

Esse tipo de interação deve propagar-se através do ligante e uma ligação direta metal-metal não é necessária. Isso porque os elétrons $d$ do íon metálico estão em orbitais $d \pi$ que podem efetivamente se recobrir com aqueles $\pi$ do ligante-ponte, tornando-os deslocalizados de uma certa extensão entre ambos os centros metálicos através do ligante. Assim, a redução de um centro metálico resulta em uma mudança de densidade eletrônica que é imediatamente comunicada ao outro sítio através da ponte, tornando mais difícil uma segunda etapa de redução.

Visto que a separação redox pode ser grande em casos onde a comunicação eletrônica é forte, então a espécie de valência mista $(\mathrm{O}, \mathrm{R})$ pode ser obtida em uma variedade de condições e também deverá ser estável com respeito ao seu desproporcionamento aos estados iniciais isovalentes oxidado $(\mathrm{O}, \mathrm{O})$ e reduzido $(\mathrm{R}, \mathrm{R})$. Normalmente, a espécie de valência mista é gerada eletroquimicamente por aplicação de potencial no domínio intermediário dos potenciais redox associados aos dois sítios metálicos (Figura 3).

Assim, a estabilidade de um complexo de valência mista hipotético, representado por $\mathrm{M}^{\mathrm{II}}-\mathrm{L}-\mathrm{M}^{\mathrm{III}}$, em relação aos seus estados isovalentes $\mathrm{M}^{\mathrm{II}}-\mathrm{L}-\mathrm{M}^{\mathrm{II}}$ e $\mathrm{M}^{\mathrm{III}}-\mathrm{L}-\mathrm{M}^{\mathrm{III}}$ é expressa pela constante de comproporcionamento ${ }^{6-8,95}, K_{\mathrm{c}}$ (Eq. 13), que é a constante de equilíbrio da reação: 


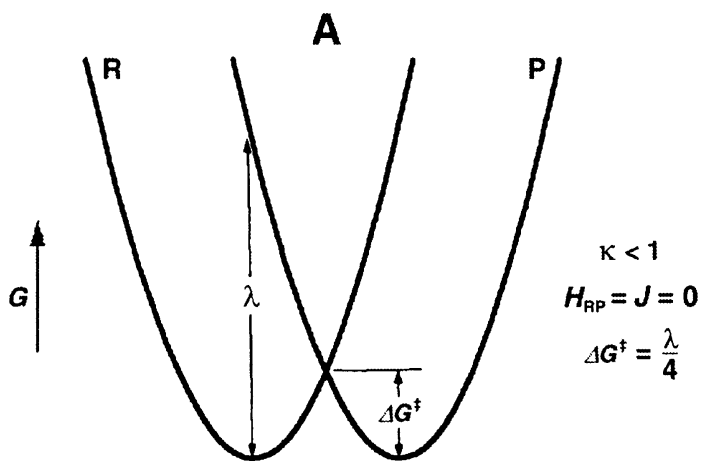

B


Figura 2. Curvas de energia potencial para reações de transferência de elétrons em sistemas de valência mista simétricos com diferentes graus de adiabaticidade: $(\boldsymbol{A})$ classe I, $(\boldsymbol{B})$ classe II e $(\boldsymbol{C})$ classe III. Nelas, $\boldsymbol{\kappa}$ é o fator de adiabaticidade ${ }^{92} ; \lambda$ é a energia de reorganização; $\mathrm{J}=\mathrm{H}_{R P}=\mathrm{H}_{a b}$ é a energia de ressonância; $e \Delta \mathrm{G}_{o} \neq$ é a energia de ativação de ordem zero, na ausência de perturbação. Cada um dos parâmetros acima será melhor definido no texto a seguir

$$
\begin{aligned}
& {\left[\mathrm{M}^{\mathrm{II}}-\mathrm{L}-\mathrm{M}^{\mathrm{II}}\right]+\left[\mathrm{M}^{\mathrm{III}}-\mathrm{L}-\mathrm{M}^{\mathrm{III}}\right]} \\
& K_{\mathrm{c}}=\frac{\left[\mathrm{M}^{\mathrm{II}}-\mathrm{L}-\mathrm{M}^{\mathrm{III}}\right]}{\left.\left[\mathrm{M}^{\mathrm{II}} \mathrm{M}^{\mathrm{II}}\right]\left[\mathrm{M}^{\mathrm{III}}\right]^{2} \mathrm{M}^{\mathrm{III}}\right]}
\end{aligned}
$$

Evidentemente, se o sistema se tratar de um complexo de valência



Potencial

Figura 3. Voltamograma cíclico ilustrativo de uma espécie binuclear genérica do tipo $M^{I I I I I}-L-M^{I I I I I}$ com interação eletrônica mensurável

mista (ou mais rigorosamente, de valência média) de classe III, então as cargas serão completamente deslocalizadas (p. ex. $\mathrm{M}^{\mathrm{II} / 2}-\mathrm{L}-$ $\left.\mathrm{M}^{\mathrm{II} / 2}\right)$ e a reação de comproporcionamento fica:

$$
\begin{aligned}
& {\left[\mathrm{M}^{\mathrm{II}}-\mathrm{L}-\mathrm{M}^{\mathrm{II}}\right]+\left[\mathrm{M}^{\mathrm{III}}-\mathrm{L}-\mathrm{M}^{\mathrm{III}}\right]} \\
& \sqrt{1} \\
& 2\left[\mathrm{M}^{\mathrm{II} / 2}-\mathrm{L}-\mathrm{M}^{\mathrm{II} / 2}\right]
\end{aligned}
$$

O valor de $K_{\mathrm{c}}$ pode variar de 4 (valor estatístico mínimo), em sistemas fracamente acoplados de classe II, a $>10^{13} \mathrm{em}$ sistemas fortemente acoplados de classe III. ${ }^{6}$

Se a interação for fraca ou moderada $\left(\Delta E_{1 / 2}<100 \mathrm{mV}\right)$, todas as três espécies $(\mathrm{O}, \mathrm{O} ; \mathrm{O}, \mathrm{R} ; \mathrm{e} \mathrm{R}, \mathrm{R})$ encontram-se presentes em solução, e o valor de $K_{c}$ deve ser obtido através de medidas espectroscópicas $^{54,55}$. Sutton e Taube têm descrito um método baseado na titulação do complexo com um agente redox ${ }^{54}$, onde a concentração máxima da espécie de valência mista é obtida indiretamente, por meio da relação de absorbância em função do número de equivalentes da espécie titulante ${ }^{6}$.

No entanto, se a interação é forte o suficiente para promover um deslocamento total do equilíbrio da Eq. 12 no sentido favorável à reação de comproporcionamento, para fins práticos somente a espécie de valência mista poderá ser monitorada e a constante de comproporcionamento, $K_{\mathrm{c}}$, poderá ser estimada diretamente através dos dados eletroquímicos ${ }^{96}$, já que $\Delta G^{0}=-\mathrm{R} T\left(\ln K_{c}\right)=-n_{1} n_{2} F(\Delta E)$ (onde $\Delta E=E_{1}-E_{2}, E_{1}$ e $E_{2}$ são os potenciais associados aos pares redox representados nas Eqs. 15 e 16, e $n_{1}$ e $n_{2}$ são os números de elétrons correspondentes a cada uma dessas etapas, respectivamente).

$$
\begin{aligned}
& {[\mathrm{O}, \mathrm{O}]^{y}+n_{1} \mathrm{e}^{-} \rightleftharpoons[\mathrm{R}, \mathrm{O}]^{y-n 1}\left(E_{1}\right)} \\
& {[\mathrm{R}, \mathrm{O}]^{y-n 1}+n_{2} \mathrm{e}^{-} \rightleftharpoons[\mathrm{R}, \mathrm{R}]^{y-n 1-n 2}\left(E_{2}\right)}
\end{aligned}
$$

Para uma reação genérica do tipo representado acima, a expressão geral de $K_{\mathrm{c}}$ é dada por: ${ }^{96}$

$K_{\mathrm{c}}=\exp \left(\frac{\left(E_{1}-E_{2}\right)\left(n_{1} n_{2}\right) F}{\mathrm{R} T}\right)$

Entretanto, para uma reação simples envolvendo 1 elétron $\left(n_{1}=\right.$ $n_{2}=1$ ), conforme esquematizado nas Eqs. 18 e 19 (que é o caso 
normalmente estudado em complexos de valência mista com metais de transição de configuração $d^{5} / d^{6}$ de spin baixo), a equação acima pode ser simplificada para resultar a Eq. 20.

$$
\begin{aligned}
& {\left[\mathrm{M}^{\mathrm{III}}-\mathrm{L}-\mathrm{M}^{\mathrm{II}}\right]^{x+1}+\mathrm{e}^{-} \rightleftharpoons\left[\mathrm{M}^{\mathrm{II}}-\mathrm{L}-\mathrm{M}^{\mathrm{III}}\right]^{x}\left(E_{1}\right)} \\
& {\left[\mathrm{M}^{\mathrm{II}}-\mathrm{L}-\mathrm{M}^{\mathrm{III}}\right]^{x}+\mathrm{e}^{-} \rightleftharpoons\left[\mathrm{M}^{\mathrm{II}}-\mathrm{L}-\mathrm{M}^{\mathrm{II}}\right]^{x-1}\left(E_{2}\right)}
\end{aligned}
$$

$$
K_{\mathrm{c}}=\exp \left(\frac{\Delta E_{1 / 2}}{25,69 \mathrm{mV}}\right) \quad(T=298 \mathrm{~K})
$$

(na equação acima, a separação $\Delta E_{1 / 2}$ deve ser dada em $\mathrm{mV}$ )

A energia livre de comproporcionamento associada à constante de equilíbrio $K_{\mathrm{c}}$ é composta de uma soma de, no mínimo, quatro termos:

$\Delta G_{\mathrm{c}}=\Delta G_{\mathrm{en}}+\Delta G_{\mathrm{el}}+\Delta G_{\mathrm{s}}+\Delta G_{\mathrm{d}}$

onde $\Delta G_{\mathrm{en}}$ é um fator entrópico que reflete a distribuição estatística da Eq. 12 (avaliado como $\left.1 / 2 \mathrm{R} T \ln ^{1 / 4} / 4\right) ; \Delta G_{\mathrm{el}}$ é um fator eletrostático oriundo da repulsão dos dois centros metálicos carregados conectados pela ponte (este termo é negligenciável em sistemas de interação moderada ou forte; em sistemas de classe II fracamente acoplados com $K_{\mathrm{c}}<30$, seu valor é tipicamente $<0,15 \mathrm{kcal} \mathrm{mol}^{-1}$, o que equivale à contribuição medíocre de $\sim 6,5 \mathrm{mV})^{6} ; \Delta G_{\mathrm{s}}$ é um fator sinergístico, oriundo da estabilização por transferência de carga e efeitos aceitadordoador de esfera externa (na maioria dos sistemas de classe II simétricos, este termo é responsável pela maior parcela na estabilização da espécie de valência mista); e o quarto termo, $\Delta G_{\mathrm{d}}$ (fator de deslocalização ou ressonância), é, na verdade, o único componente da energia de estabilização com origem na deslocalização eletrônica (este é o termo dominante em sistemas de classe III).

Para sistemas fracamente acoplados, a magnitude de $\Delta G_{\mathrm{d}}$ (Eq. $22)^{6}$ pode ser estimada a partir da energia de Franck-Condon, $\lambda$, e da energia de ressonância, $H_{\mathrm{ab}}$, que, por sua vez, pode ser extraída dos parâmetros da banda de intervalência (v. mais adiante). $\Delta G_{\mathrm{d}}$ é comumente de $\sim 1 \%$ do valor de $H_{\mathrm{ab}}$ para a maioria dos sistemas de cargas localizadas reportados na literatura ${ }^{6}$.

$\Delta G_{\mathrm{d}}=\frac{H_{\mathrm{ab}}^{2}}{\lambda}$

Em sistemas totalmente deslocalizados, $\Delta G_{\mathrm{d}}$ é estimado por:

$\Delta G_{\mathrm{d}} \approx F \times \Delta E_{1 / 2} \approx H_{\mathrm{ab}} \approx 1 / 2 E_{\mathrm{op}}$

A natureza do ligante-ponte desempenha um papel bastante importante na intensidade da comunicação eletrônica, especialmente através de interações de transferência de carga combinadas do tipo $\pi$ (ligante) $\rightarrow d \pi$ (metal) e do tipo $d \pi$ (metal) $\rightarrow \pi^{*}$ (ligante). Ligantes de pequena dimensão molecular são mais eficientes que os de maiores dimensões e, para um dado ligante-ponte, a natureza eletrônica do íon metálico e dos coligantes também pode exercer uma influência dramática sobre a magnitude do acoplamento eletrônico, cuja determinação é o assunto da próximo item.

\section{Acoplamento Eletrônico e Deslocalização}

O exemplo mais simples de molécula de valência mista talvez seja o cátion de hidrogênio molecular, $\mathrm{H}_{2}{ }^{+}$. Nesse caso, a interação entre as espécies doadora e aceitadora se dá por meio de recobrimento orbital direto, promovendo a deslocalização da carga que, nesse exemplo em particular, é responsável pela ligação química.

Contudo, na maioria dos complexos de valência mista inorgânicos, os centros metálicos encontram-se separados por uma espécie conectora intermediária (chamada de ponte ou ligante-ponte) a uma distância suficientemente grande para promover uma perturbação fraca ou moderada com localização de cargas. Nesse caso, o recobrimento orbital entre os centros doador e aceitador é desprezível e a interação eletrônica se propaga através do ligante-ponte, principalmente por meio de conjugação orbital do tipo $\pi^{97}$.

Se existirem estados redox localizados, o elétron ímpar se comportará como uma carga em oscilação contínua através do liganteponte. A Eq. 24 ilustra esse fenômeno para um complexo binuclear de valência mista formado pela associação de íons do tipo $\mathrm{M}^{\mathrm{III}} / \mathrm{M}^{\mathrm{II}}$ $\left(d^{5} / d^{6}\right.$ de spin baixo).

$\mathrm{M}^{\mathrm{III}}-\mathrm{L}-\mathrm{M}^{\mathrm{II}} \stackrel{k_{\mathrm{ET}}}{\rightleftharpoons} \mathrm{M}^{\mathrm{II}}-\mathrm{L}-\mathrm{M}^{\mathrm{III}}$

A medida direta da constante de velocidade de troca eletrônica intermetálica (Eq. 25) pode ser muito difícil. Entretanto, o análogo óptico (transferência induzida por luz; Eq. 26) normalmente pode ser observado tanto em sistemas simétricos $\left(\mathrm{M}_{\mathrm{a}}=\mathrm{M}_{\mathrm{b}} ; \Delta G^{0}=0\right)$ como em assimétricos $\left(\mathrm{M}_{\mathrm{a}} \neq \mathrm{M}_{\mathrm{b}} ; \Delta G^{0} \neq 0\right)$.

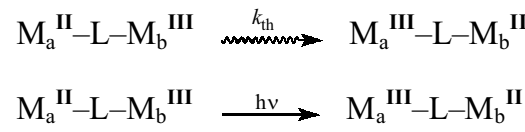

O processo da Eq. 26 também pode ser representado pela transição eletrônica $\mathrm{M}_{\mathrm{a}}(d \pi) \rightarrow(d \pi) \mathrm{M}_{\mathrm{b}}$ relacionada à transferência de intervalência, conforme explicitado abaixo:

$\mathrm{M}_{\mathrm{a}}{ }^{\mathrm{II}}\left(d \pi_{3}{ }^{2}\right)-\mathrm{L}-\left(d \pi_{3}{ }^{1}\right) \mathrm{M}_{\mathrm{b}}{ }^{\mathrm{II}} \stackrel{\mathrm{h} v}{\longrightarrow} \mathrm{M}_{\mathrm{a}}{ }^{\mathrm{III}}\left(d \pi_{3}{ }^{1}\right)-\mathrm{L}-\left(d \pi_{3}{ }^{2}\right) \mathrm{M}_{\mathrm{b}}{ }^{\mathrm{II}}$

onde $d \pi_{3}$ é o nível de energia $d \pi$ de maior energia entre os três orbitais metálicos do tipo $t_{2 \mathrm{~g}}$ quase degenerados ${ }^{98}$.

Em complexos de interação muito forte (classe III de acordo com a classificação de Robin e Day) ${ }^{3}$ o elétron ímpar do estado de valência mista está completamente deslocalizado entre os metais, e uma descrição como [II $\left.1 \frac{1}{2}, \mathrm{II} 1 \frac{1}{2}\right]$ é mais apropriada que [II,III] para ambos os estados de oxidação. Nesse caso, a promoção eletrônica do estado fundamental para um estado de maior energia (processo radiativo) será efetivamente uma transição $\pi \rightarrow \pi^{*}$ (Eq. 28).

$\mathrm{M}_{\mathrm{a}}{ }^{\mathrm{II} / 2}-\mathrm{L}-\mathrm{M}_{\mathrm{b}}{ }^{\mathrm{II} / 2} \stackrel{h \mathrm{v}}{\longrightarrow}\left[\mathrm{M}_{\mathrm{a}}{ }^{\mathrm{II} / 2}-\mathrm{L}-\mathrm{M}_{\mathrm{b}}{ }^{\mathrm{II} / 2}\right]^{*}$

No entanto, em complexos onde a interação entre os metais é moderada (classe II), as valências são localizadas e uma descrição [II,III] se aplica. Nesse caso, existe a possibilidade de um elétron ser transferido do metal de menor estado de oxidação para o outro sítio metálico de maior estado de oxidação (Eqs. 26 e 27).

Esse tipo especial de transferência de carga intramolecular foi chamado de transferência de intervalência (IT) por Hush, mas é comum encontrar na literatura atribuição do mesmo fenômeno à transferência de carga metal-metal (MMCT).

\section{Modelo de Hush}

O objetivo deste tópico é fornecer uma visão geral da teoria perturbacional de $\mathrm{Hush}^{5,59,60}$ e introduzir as suas principais equações e relações. No caso de interesse em uma abordagem mais aprofundada do formalismo envolvido, o leitor poderá consultar o Apêndice, onde se encontra um resumo do desenvolvimento do modelo, que é base- 
ado no formalismo de Mulliken para complexos de transferência de $\operatorname{carga}^{100,101}$.

Em um complexo de valência mista do tipo $\mathrm{M}^{\mathrm{II}}-\mathrm{L}-\mathrm{M}^{\mathrm{III}}$ (com cargas localizadas), a interação eletrônica entre o doador $\left(\mathrm{M}^{\mathrm{II}}\right)$ e o aceitador $\left(\mathrm{M}^{\mathrm{III}}\right)$ através do ligante-ponte mistura os caráteres eletrônicos e induz a transferência eletrônica. Essa comunicação também cria uma base eletrônica para a indução da transferência óptica dipolopermitida, com a magnitude da perturbação refletindo sobre a intensidade da banda de transferência de carga metal-metal. Para uma banda com formato Gaussiano em um sistema de dois níveis, a energia de ressonância $\left(H_{\mathrm{ab}}\right)$ originada pelo acoplamento eletrônico e o respectivo grau de deslocalização eletrônica $\left(\alpha^{2}\right)$ podem ser calculados a partir das Eqs. 29 e 30, respectivamente.

$H_{\mathrm{ab}}=2,05 \times 10^{-2}\left(\frac{\varepsilon_{\text {máx }} \cdot \bar{v}_{\text {máx }} \cdot \Delta \bar{v}_{1 / 2}}{d^{2}}\right)^{1 / 2}$

$\alpha^{2}=\left(\frac{H_{\mathrm{ab}}}{E_{\mathrm{op}}}\right)^{2}$

onde os parâmetros envolvidos são: $\bar{v}_{\text {máx }}=E_{\text {op }}$, a energia da absorção máxima; $\varepsilon_{\text {máx }}$, a absortividade molar; e $\Delta \bar{v}_{1 / 2}$, a largura da banda à metade de sua altura/intensidade, que são obtidos diretamente a partir da banda associada à transferência de intervalência (v. banda de absorção ilustrativa na Figura 4); e $d$, a distância de transferência eletrônica (que é usualmente assumida como sendo equivalente à separação metal-metal $)^{102}$.

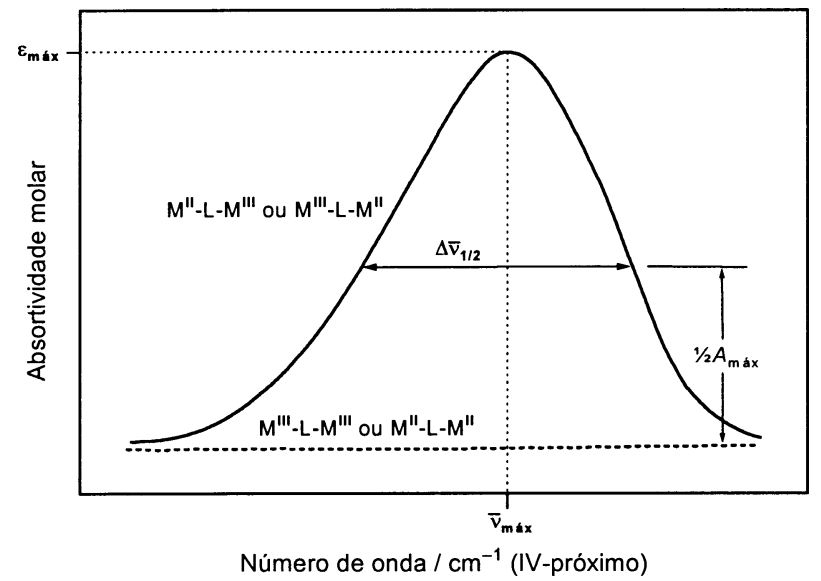

Figura 4. Espectros de absorção eletrônica típicos de espécies isovalentes $M^{I I I}-L-M^{I I I}$ e $M^{I I}-L-M^{I I}(\ldots .$.$) e de valência mista M^{I I}-L-M^{I I I}$ ou $M^{I I I}-L-M^{I I}$ (一). A banda associada à transferência de intervalência ilustra alguns dos parâmetros empregados na estimativa do elemento de matriz do acoplamento eletrônico, $\mathrm{H}_{a b}$

Outros formalismos teóricos mais rigorosos do ponto de vista quântico encontram-se disponíveis atualmente ${ }^{88-90}$. Todavia, a teoria de Hush ainda parece apresentar mais vantagens e maior aplicabilidade para sistemas com acoplamento moderado que os demais, o que a transforma no modelo mais adotado pelos cientistas no âmbito das interações eletrônicas doador-aceitador.

\section{Algumas Relações de Marcus-Hush}

A Figura 5 ilustra algumas relações energéticas em um processo de transferência de intervalência. Nessas curvas são utilizadas coor- denadas generalizadas que incluem as vibrações e as oscilações acopladas do solvente, que são tratadas classicamente como um conjunto de osciladores harmônicos.

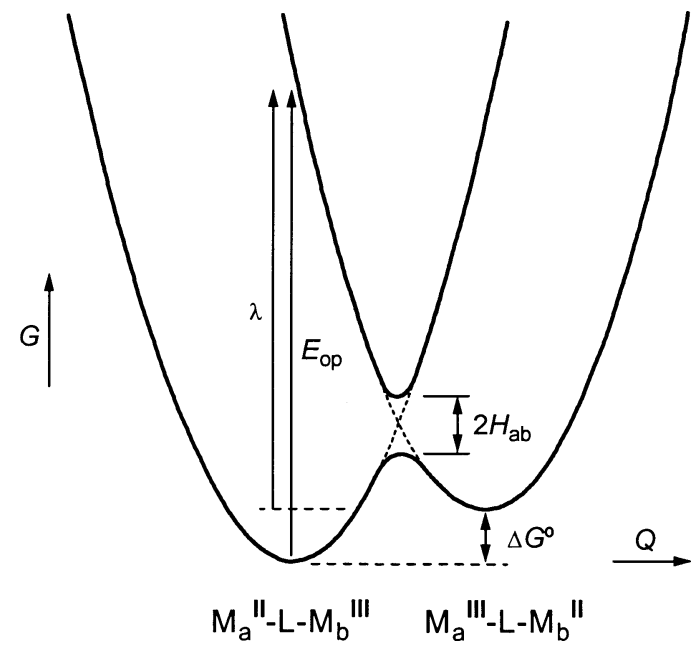

Figura 5. Diagramas de energia vs. coordenadas nucleares para uma transferência eletrônica adiabática e assimétrica ${ }^{104}$

Algumas relações importantes entre transferência de elétrons térmica e óptica podem ser derivadas diretamente por meio dessas curvas $^{105}$. A energia de transferência de elétrons óptica $\left(v_{\text {máx }}\right.$ ou $\left.E_{\mathrm{op}}\right)$, por exemplo, é ditada pela energia de reorganização ${ }^{62}$ do sistema $(\lambda),{ }^{108}$ que, por sua vez, é uma função de suas componentes de esferas interna $\left(\lambda_{i}\right)$ e externa $\left(\lambda_{\mathrm{o}}\right)$. Para os casos simétrico e assimétrico, tal relação é expressa pelas Eqs. 31 e 32, respectivamente.

$$
\begin{aligned}
& \bar{v}_{\text {máx }}=\lambda=\lambda_{\mathrm{i}}+\lambda_{\mathrm{o}} \\
& \bar{v}_{\text {máx }}=\Delta G^{\mathrm{o}}+\lambda=\Delta G^{\mathrm{o}}+\lambda_{\mathrm{i}}+\lambda_{\mathrm{o}}
\end{aligned}
$$

onde $\lambda_{\mathrm{i}}$ e $\lambda_{\mathrm{o}}$ são dadas pelas Eqs. 33 e 34 a seguir.

$$
\begin{aligned}
& \lambda_{\mathrm{i}}=\sum_{n}\left(\frac{2 f_{\mathrm{R}} f_{\mathrm{P}}}{f_{\mathrm{R}}+f_{\mathrm{P}}}\right)_{n}\left(q_{\mathrm{R}}-q_{\mathrm{P}}\right)_{n}^{2} \\
& \lambda_{\mathrm{o}}=(\Delta e)^{2}\left(\frac{1}{2 r_{\mathrm{D}}}+\frac{1}{2 r_{\mathrm{A}}}-\frac{1}{r_{D A}}\right)\left(\frac{1}{\varepsilon_{\mathrm{op}}}-\frac{1}{\varepsilon_{\mathrm{s}}}\right)
\end{aligned}
$$

Nas equações acima, $f_{\mathrm{R}}$ e $f_{\mathrm{P}}$ são as constantes de força para o $n$ ésimo modo vibracional das ligações metal-ligante no reagente e no produto; $\left(q_{\mathrm{R}}-q_{\mathrm{P}}\right)$ é a variação de distância e ângulo de ligação que acompanha a transformação do reagente em produto; $r_{\mathrm{D}} \mathrm{e} r_{\mathrm{A}}$ são os raios iônicos dos reagentes doador e aceitador; $r_{\mathrm{DA}}$ é a distância de contato $\left(r_{\mathrm{D}}+r_{\mathrm{A}}\right)$; e $\varepsilon_{\mathrm{op}}$ e $\varepsilon_{\mathrm{s}}$ são as constantes dielétricas óptica (correspondente à polarização eletrônica do meio; $\varepsilon_{\text {op }}=\eta^{2}$ ) e estática (permissividade relativa correspondente ao dipolo do solvente).

O modelo acima assume uma coordenação esférica onde cada um dos sítios (D e A) é representado pelo centro de sua esfera iônica. Além desse, um modelo elipsoidal também foi proposto por Cannon ${ }^{109}$, onde se acredita que os elipsóides representem melhor o formato/volume molecular do sistema doador-aceitador. Ambos os modelos são fundamentados na aproximação do contínuo dielétrico; isto é, assumem uma dependência homogênea das propriedades físico-químicas do solvente $\left(\varepsilon_{\mathrm{op}}^{-1}-\varepsilon_{\mathrm{s}}^{-1}\right)$. 
Dessa forma, as transições de intervalência têm sido bastante úteis na exploração de efeitos estruturais e de solvente em reações de transferência de elétrons. Por exemplo, nos complexos simétricos $\left[\mathrm{Cl}(\text { bpy })_{2} \mathrm{Ru}^{\mathrm{III}}(\mathrm{L}) \mathrm{Ru}^{\mathrm{II}}(\mathrm{bpy})_{2} \mathrm{Cl}\right]^{3+}(\mathrm{L}=$ pirazina, 4,4'-bipiridina, 1,2bis(4-piridil)etileno), $d$ aumenta de 6,8 para $13,8 \AA$ através da série. Nela, observou-se que $E_{\text {op }}$ (IT) varia com $1 / \varepsilon_{\text {op }}-1 / \varepsilon_{\mathrm{s}}$ de acordo com as Eqs. 31 a 34 em uma série de solventes orgânicos polares e aumenta com $1 / d$ em $\mathrm{CD}_{3} \mathrm{CN}^{6,110}\left(\bar{v}_{\text {máx }}\right.$ aumenta de 7700 a $10800 \mathrm{~cm}^{-1}$ e $H_{\text {ab }}$ diminui de $400 \mathrm{a} \sim 150 \mathrm{~cm}^{-1}$, no mesmo sentido). Ambos os efeitos são preditos pelo contínuo dielétrico resultante, assumindo duas esferas sem interpenetração. Melhor concordância foi obtida com um modelo de cavidade elipsoidal ${ }^{111}$.

Entretanto, é importante mencionar que, devido à aproximação do contínuo dielétrico, essa teoria funciona adequadamente para sistemas relativamente hidrofóbicos na ausência de interações específicas do tipo soluto-solvente (p. ex. em complexos polipiridil-derivados) e/ ou simétricos ${ }^{6}$, mas ela falha em complexos assimétricos contendo solvatação preferencial (p. ex. amina ${ }^{7,112}$, onde ligações/pontes de hidrogênio podem ocorrer, e ciano-derivados ${ }^{113}$, onde a doação de um par eletrônico resulta em interações doador-aceitador). Isso porque os ligantes de cada fragmento terminal possuem interações específicas com moléculas individuais do solvente, podendo, portanto, dar origem a uma série de fenômenos ${ }^{114}$. Por exemplo, a unidade metálica contendo o sítio oxidado $\left(\mathrm{Ru}^{\text {III }}\right)$ em $\left[\left(\mathrm{NH}_{3}\right)_{5} \mathrm{Ru}^{\mathrm{III}}\left(4,4^{\prime}-\text {-bpy }\right) \mathrm{Ru}^{\mathrm{II}}\left(\mathrm{NH}_{3}\right)_{5}\right]^{5+}$ é seletivamente solvatada por dimetilsulfóxido em mistura de dimetilsulfóxido:acetonitrila. Isso maximiza a ligação de hidrogênio sobre o íon $\mathrm{Ru}^{\mathrm{III}}$, que é o mais ácido ${ }^{115}$. Em [Cl(bpy) ${ }_{2} \mathrm{Os}^{\mathrm{III}}(4,4$ ' bpy) $\left.\mathrm{Ru}^{\mathrm{II}}\left(\mathrm{NH}_{3}\right)_{5}\right]^{4+}$, os potenciais redox para $\mathrm{Os}^{\mathrm{III/II}}$ e $\mathrm{Ru}^{\mathrm{II} / \mathrm{II}}$ são muito próximos devido à natureza distinta dos ligantes presentes em cada centro. Dessa maneira, a escolha apropriada do solvente pode provocar a transferência eletrônica dirigida $\mathrm{Ru}^{\mathrm{II}} \rightarrow \mathrm{Os}^{\mathrm{III}}$, por otimização das interações do solvente com $\left\{-\mathrm{Ru}^{\mathrm{III}}\left(\mathrm{NH}_{3}\right)_{5}\right\}^{3+}{ }^{112}$ Nesses casos, não há correlação definida entre $E_{\mathrm{op}} \mathrm{e}\left(\varepsilon_{\mathrm{op}}{ }^{-1}-\varepsilon_{\mathrm{s}}^{-1}\right)$, sendo recomendável a utilização dos parâmetros DN (donor number) ou AN (acceptor number) de Gutmann ${ }^{116}$ na racionalização dos efeitos solvatocrômicos.

Embora muitas análises de constantes de velocidade de transferência de elétrons tenham demonstrado uma correlação evidente com a variação de parâmetros estruturais de esfera interna (tal como distância de ligação metal-ligante) ${ }^{117}, \lambda_{\mathrm{i}}$ só se torna bastante relevante quando ocorrem mudanças geométricas drásticas com a transferência de elétrons, como mudança de simetria molecular ou de número de coordenação; ou então quando a reação se dá em solventes apolares (nos quais a contribuição de $\lambda_{\mathrm{o}}$ é aproximadamente nula). Em solventes polares, $\lambda_{\mathrm{o}}$ é tipicamente da ordem de 1,0-1,5 eV, dominando totalmente a componente $\lambda_{\mathrm{i}}$, cujo valor típico em complexos de valência mista de configuração $d^{5} / d^{6}$ de spin baixo se insere na faixa de $0,1-0,3 \mathrm{eV}^{6}$

Evidentemente, a localização/deslocalização de cargas sobre o sistema de valência mista também exerce um papel determinante na contribuição de cada um dos termos $\lambda_{\mathrm{i}}$ e $\lambda_{\mathrm{o}}$. Esse aspecto, porém, será enfatizado adiante junto com os critérios para se distinguir sistemas de classe II ou classe III.

As expressões dadas nas Eqs. 31 e 32 para $\bar{v}_{\text {máx }}$ estão relacionadas às correspondentes energias de ativação para um processo de transferência de elétrons térmico $\left(\Delta G^{\ddagger} \approx \Delta G_{o}^{\ddagger}-H_{a b}\right)$ por:

$$
\begin{aligned}
& \Delta G_{\mathrm{o}}^{\ddagger}=\frac{\bar{v}_{\text {máx }}}{4}=\frac{\lambda}{4}\left(\Delta G^{\mathrm{o}}=0\right) \\
& \Delta G_{\mathrm{o}}^{\ddagger}=\frac{\left(\bar{v}_{\text {máx }}\right)^{2}}{4\left(\bar{v}_{\text {máx }}-\Delta G^{\mathrm{o}}\right)}=\frac{\left(\lambda+\Delta G^{\mathrm{o}}\right)^{2}}{4 \lambda}\left(\Delta G^{\mathrm{o}} \neq 0\right)
\end{aligned}
$$

E a constante de velocidade de transferência de elétrons é:

$k_{\mathrm{ET}}=\kappa v_{\mathrm{n}} \exp \left(\frac{-\Delta G^{\ddagger}}{R T}\right) \approx v_{\mathrm{n}} \exp \left(-\frac{\left(\Delta G_{\mathrm{o}}^{\ddagger}-H_{a b}\right)}{R T}\right)$

Nesses sistemas, a largura de meia-banda teórica é dada por:

$$
\left(\Delta \bar{v}_{1 / 2}\right)^{2}=16\left(\bar{v}_{\text {máx }}-\Delta G^{\mathrm{o}}\right) R T \ln 2
$$

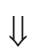

$\Delta \bar{v}_{1 / 2}=(16 \lambda R T \ln 2)^{1 / 2}$

Nas relações acima, as mudanças de freqüência nas vibrações acopladas e nas oscilações do solvente ( $\left.\hbar \omega \cong \hbar \omega^{\prime}\right)$ estão incluídas em $\Delta G^{\mathrm{o}}$ como uma contribuição para a diferença entrópica entre es$\operatorname{tados}\left(\operatorname{se} \Delta \omega=\left|\omega-\omega^{\prime}\right|<<\omega, \omega^{\prime}\right)^{118}$.

Assim sendo, uma das razões do grande interesse na química dos complexos de valência mista é o possível uso de medidas espectrais simples para se determinar barreiras de energia e, por conseguinte, calcular constantes de velocidade de transferência de elétrons térmica, $k_{\mathrm{ET}}$, através dos parâmetros ópticos da banda de intervalência. Deste modo, $\Delta G^{\ddagger}$ e $\lambda$ são obtidos do máximo da banda e da largura de meia-banda, e $H_{\mathrm{ab}}$ é calculado da intensidade de banda integrada. Isso permite comparar constantes de velocidade de transferência de elétrons teórica e experimental, um assunto de fundamental importância que vem se destacando em várias áreas da química $^{119}$.

A aproximação clássica funciona razoavelmente bem para vibrações de baixa frequiência, tal como o estiramento metal-ligante na temperatura ambiente (ou no caso em que $\hbar \omega \sim k_{\mathrm{B}} T$ ). Entretanto, na presença de modos que são correspondentes a anéis aromáticos ou vibrações $\mathrm{CO}$ e $\mathrm{CN}$, nos quais $\hbar \omega \gg k_{\mathrm{B}} T$, deve-se incluir esta contribuição explicitamente na barreira vibracional, uma vez que, neste caso, a aproximação clássica não se aplica.

Em complexos de valência mista simétricos, as bandas de intervalência são tipicamente encontradas na região do infravermelho próximo (de 1000 a $2000 \mathrm{~nm}$, ou de 5000 a $10000 \mathrm{~cm}^{-1}$ ). Elas podem aparecer em energias maiores para complexos assimétricos, já que $\bar{v}_{\text {máx }}$ depende de $\Delta G^{\mathrm{o}}$. Além disso, essas bandas normalmente são largas e dependentes do solvente, com absortividade molar variando de 10 a $10^{4} \mathrm{~mol}^{-1} \mathrm{dm}^{3} \mathrm{~cm}^{-1}$, dependendo do ligante-ponte. Ligantes pequenos com níveis $\pi$ ou $\pi^{*}$ acessíveis, tais como moléculas diatômicas (p. ex. $\mathrm{O}^{2-}, \mathrm{N}_{2}, \mathrm{CN}^{-}$) e anéis heterocíclicos simples conjugados (p. ex. pirazina), promovem acoplamento eletrônico mais forte por mistura do caráter ligante nos orbitais $d \pi$ do metal.

\section{Distinção entre Classe II e Classe III}

Basicamente, existem dois critérios práticos para se "tentar" distinguir entre sistemas de classes II e III: (a) análise da largura de meia-banda associada à banda de intervalência, e (b) investigação da dependência de $\bar{v}_{\text {máx }}$ com o solvente.

O primeiro critério (a) foi proposto por Hush e decorre diretamente das premissas do seu modelo perturbacional: se o sistema possui cargas localizadas (perturbação fraca, de primeira ordem) então a largura de meia-banda teórica pode ser estimada através da Eq. 38, que, à $T=298 \mathrm{~K}$, resulta as Eqs. 39 e 40 para sistemas simétrico e assimétrico, respectivamente.

$\Delta \bar{v}_{1 / 2}=\left(2310 \cdot \bar{v}_{\text {máx }}\right)^{1 / 2}$ 
$\Delta \bar{v}_{1 / 2}=\left[2310 \cdot\left(\bar{v}_{\text {máx }}-\Delta G^{\mathrm{o}}\right)\right]^{1 / 2}$

De acordo com essa proposição, o valor obtido dessa forma estaria no limite da transição carga localizada/carga deslocalizada. Assim, se o valor obtido experimentalmente for superior ou igual ao previsto pelas Eqs. 39 e 40, então o sistema é de classe II e a teoria de Hush se aplica. Se, por outro lado, $\Delta \bar{v}_{1 / 2}$ (experimental) $<\Delta \bar{v}_{1 / 2}$ (calculado), então o sistema se enquadra melhor na classe III de Robin-Day, com descrição de cargas deslocalizadas. Nesse caso, o modelo de Hush não se aplica adequadamente; todavia, ainda assim é empregado freqüentemente para fins comparativos ${ }^{6,9}$.

O segundo critério (b) deriva do fato de que, sendo um sistema de cargas deslocalizadas (classe III), não haverá uma dependência entre energia de transferência de elétrons óptica $\left(\bar{v}_{\text {máx }}\right)$ e energias de reorganização $\left(\lambda_{i}\right.$ e $\left.\lambda_{o}\right)$, conforme indicado nas relações de HushMarcus. Nesse caso, tanto as variações de esfera interna como a reorientação de dipolo do solvente ao redor dos centros redox deixam de ser importantes na escala de tempo das transformações nucleares, uma vez que todos os modos se tornam médios/simétricos $\left(\mathrm{M}_{\mathrm{a}}-\mathrm{L} \equiv \mathrm{M}_{\mathrm{b}}-\mathrm{L}\right)$ e $\lambda_{\mathrm{i}}$ não é afetado. Da mesma forma, assumindo deslocalização eletrônica completa, não haverá variação líquida de carga entre $\mathrm{M}_{\mathrm{a}}{ }^{\mathrm{II} / 2} \mathrm{e} \mathrm{M}_{\mathrm{b}}{ }^{\mathrm{II}}$, , de tal forma que a reorganização do solvente se torna irrelevante/invariante.

As idéias acima podem ser muito bem ilustradas através da Figura $2 \mathrm{C}$, onde os estados reagente e produto se encontram verticalmente alinhados devido à grande estabilização por ressonância (no limite onde $E_{\mathrm{op}}=2 H_{\mathrm{ab}}$ ). Isso significa que a transferência de carga não requer rearranjo nuclear.

Uma comparação prática é dada pelos dímeros análogos $\left[\left\{\left(\mathrm{NH}_{3}\right)_{5} \mathrm{Ru}\right\}_{2}(\mathrm{pz})\right]^{5+14}$ e $\left[\left\{\left(\mathrm{NH}_{3}\right)_{5} \mathrm{Ru}\right\}_{2}\left(4,44^{\prime}-\text { bpy }\right)\right]^{5+}$ 53-55: enquanto o primeiro possui cargas deslocalizadas $\left(\operatorname{com} K_{\mathrm{c}}=4 \times 10^{6}, H_{\mathrm{ab}}=\right.$ $3300 \mathrm{~cm}^{-1}, \bar{v}_{\text {máx }}=6400 \mathrm{~cm}^{-1}, \varepsilon_{\text {máx }}=5000 \mathrm{~mol}^{-1} \mathrm{dm}^{3} \mathrm{~cm}^{-1}, \Delta \bar{v}_{1 / 2}=$ $1200 \mathrm{~cm}^{-1}$, e $\left.d=6,8 \AA\right)^{6}$ e $E_{\text {op }}$ praticamente independe do solvente, o segundo apresenta comunicação eletrônica fraca entre os seus centros metálicos com cargas localizadas (os parâmetros obtidos em $\mathrm{D}_{2} \mathrm{O}$ são: $K_{\mathrm{c}}=20, H_{\mathrm{ab}}=390 \mathrm{~cm}^{-1}, \bar{v}_{\text {máx }}=9700 \mathrm{~cm}^{-1}, \varepsilon_{\text {máx }}=920 \mathrm{~mol}^{-1} \mathrm{dm}^{3} \mathrm{~cm}^{-1}$, e $\left.\Delta \bar{v}_{1 / 2}=5200 \mathrm{~cm}^{-1} ; d=11,3 \AA\right)^{6}$ e é solvatocrômico conforme o esperado pela Eq. 34 .

Outro exemplo bastante contrastante envolve algumas espécies de valência mista simétricas do tipo $\left[\left\{\mathrm{Cl}(\mathrm{bpy})_{2} \mathrm{M}\right\}_{2}(\mathrm{pz})\right]^{3+}$. Conforme mencionado anteriormente, no complexo com $\mathrm{M}=\mathrm{Ru}$, os dois centros metálicos encontram-se fracamente acoplados eletronicamen$\mathrm{te}^{6,110}\left(\left[\mathrm{Cl}(\mathrm{bpy})_{2} \mathrm{Ru}^{\mathrm{III}}(\mathrm{pz}) \mathrm{Ru}^{\mathrm{II}}(\mathrm{bpy})_{2} \mathrm{Cl}\right]^{3+}\right.$; classe II). Diferentemente, no caso do complexo análogo com $\mathrm{M}=\mathrm{Os}$, o ligante compacto e a maior extensão radial dos orbitais $5 d$ do ósmio conferem uma interação intermetálica mais forte. ${ }^{41} \mathrm{Na}$ espécie $\mu$-pirazinabis \{pentaaminósmio $\}(5+)$, em particular, verificou-se uma total deslocalização eletrônica ${ }^{120}\left(\left[\left(\mathrm{NH}_{3}\right)_{5} \mathrm{Os}^{\mathrm{II} / 2}(\mathrm{pz}) \mathrm{Os}^{\mathrm{II} / 2}\left(\mathrm{NH}_{3}\right)_{5}\right]^{5+}\right.$; classe III).

Embora essa distinção entre sistemas de classe II e de classe III pareça simples e inequivocada (e realmente o é em sistemas típicos de cargas totalmente localizadas ou completamente deslocalizadas), ela pode se tornar uma tarefa bastante árdua quando se trata de sistemas com comportamento ambíguo, no limiar das classes II e III (na maioria das vezes classificado como sistemas na fronteira das classes II/III) $)^{9,18,22}$, ou mesmo quando se trata de um sistema com características de classe III. ${ }^{9,121} \mathrm{O}$ íon $\left[\left(\mathrm{NH}_{3}\right)_{5} \mathrm{Ru}(\mathrm{pz}) \mathrm{Ru}\left(\mathrm{NH}_{3}\right)_{5}\right]^{5+}$, por exemplo, parece possuir cargas totalmente deslocalizadas (classe III), mas é freqüentemente dado como um sistema de comportamento intermediário, na situação limite classe II/classe III, ou até mesmo de classe II (dependendo da técnica utilizada na caracterização). A sua classificação tem sido um tema de muita controvérsia na literatu$\mathrm{ra}^{6,7,121}$.
O desenvolvimento de modelos adequados para se elucidar o que se classifica como transição de comportamento localizadodeslocalizado em alguns sistemas é, na verdade, um tema importante, bastante explorado e que permanece longe de estar esgotado em química de valência mista. Diferentemente do que ocorre nos casos tipicamente localizados ou deslocalizados, as ferramentas teóricas disponíveis não explicam adequadamente as propriedades intrincadas e originais desse tipo de sistemas. Com o avanço dos métodos de síntese e das técnicas físico-químicas, os resultados de estudos recentes $^{41}$ têm revelado que tais sistemas são bem mais complexos do que se imaginava. Esse aspecto da química de valência mista tem sido reconhecido como um dos mais importantes atualmente no contexto dos processos de transferência eletrônica.

\section{CONSIDERAÇÕES FINAIS}

Embora as transições de intervalência consistam essencialmente em processos de transferência eletrônica intramolecular, o conhecimento de seus mecanismos envolvidos em processos adiabáticos e não-adiabáticos pode ser útil na investigação de reações de transferência de elétrons intermolecular. Isso nos conduz à principal razão de se estudar complexos binucleares de valência mista.

Reações de transferência eletrônica a longa distância são de considerável importância em muitas áreas de química, variando desde bioinorgânica até dispositivos eletrônicos moleculares artificiais. Contudo, em muitos casos, a transferência de elétrons entre moléculas é dependente de uma série de fatores que não podem ser conhecidos detalhadamente, tais como a separação entre os centros redox no ponto de transferência e a conformação molecular, entre outros. Por outro lado, em complexos de valência mista a transferência eletrônica ocorre dentro da unidade molecular, para a qual a distância entre os metais, bem como a estrutura eletrônica e conformação molecular do ligante-ponte são bem conhecidos. Sendo assim, um processo de transferência de intervalência em uma molécula de valência mista é um modelo natural para uma reação de transferência eletrônica intra- ou intermolecular, e a informação a respeito do processo de transferência de intervalência é bastante relevante para outros processos com transferência de elétron(s). Além disso, análise da interação eletrônica nesses complexos permite comparar a habilidade de determinados ligantes atuarem como fios moleculares (dependendo da capacidade condutora destes materiais) para um potencial emprego em dispositivos eletrônicos.

Este campo de pesquisa emergiu em duas direções básicas. Primeiro, os princípios delineados acima têm sido estendidos a uma ampla variedade de unidades metálicas terminais conectadas por ligantes-ponte dos mais variados tipos. Isso proporcionou uma melhor compreensão da dependência da interação eletrônica com a natureza de diferentes ligantes e espécies metálicas. Segundo, tentativas estão sendo realizadas no sentido de se controlar essas interações a longa distância por métodos de manipulação externa, tais como: controle conformacional e dimensional do ligante-ponte ${ }^{8,60,122,123}$; controle eletroquímico externo dos estados redox ${ }^{8,31,124}$ (em sistemas onde o ligante é eletroquimicamente ativo); controle por mecanismos de isomerização fotoquímica ${ }^{125}$; ou ainda, controle e indução da interação metal-metal por meio de efeitos ácido-base ou de $\mathrm{pH}^{8,126,127}$ Além disso, transições de carga de intervalência (envolvendo transferência eletrônica vetorial em complexos de valência mista) são solvatocrômicas, pois o grau de reorganização da esfera de coordenação (que inclui solvatação) requerida nos dois sítios durante a transferência de carga é dependente do solvente. Dessa forma, os efeitos de ambiente (principalmente os do solvente) proporcionam uma outra forma natural de se modular interações metal-metal ${ }^{8,111,112,128}$.

Portanto, tais complexos bi- ou polinucleares de valência mista constituem alguns exemplos protótipos de chaves ou interruptores 
moleculares ${ }^{123,129}$, cuja elaboração é o primeiro passo na construção de dispositivos eletrônicos moleculares, uma vez que um circuito integrado consiste essencialmente de um grande número de chaves.

Entre os vários tipos de dispositivos e componentes que desempenham funções eletrônicas em escala molecular, os fios moleculares são de fundamental importância, pois podem operar como conectores que controlam/medeiam o fluxo eletrônico entre os diferentes elementos de um sistema eletrônico molecular ${ }^{8,130-132}$. Os fios moleculares permitem tanto a transferência eletrônica a longas distâncias quanto a interação/perturbação dos componentes afastados.

Muito do atual conhecimento sobre condução eletrônica em fios moleculares é baseado nas informações acumuladas nos últimos 40 anos de trabalho sobre transferência eletrônica em moléculas do tipo doador-aceitador. Dessa forma, é conhecido que o acoplamento eletrônico através de uma cadeia ligando um doador e um aceitador eletrônico pode determinar a velocidade de transferência eletrônica entre os fragmentos terminais da molécula. Esse mesmo tipo de interação mediada por ponte é reconhecido como um fator crítico na transferência de elétrons a longas distâncias em muitas estruturas biológicas (p. ex. proteínas redox modificadas ${ }^{81}$, centros de reações fotossintéticas ${ }^{74,79,133}$ e ácidos nucléicos/DNA ${ }^{83}$ ), na condução eletrônica e/ou transporte de carga por meio de camadas moleculares ${ }^{132,134-137}$ e em sistemas organometálicos ${ }^{138}$ ou eletroquímicos ${ }^{84,135,139}$, na emissão de elétrons em certas classes de emissores ${ }^{137,140}$, em processos redox próton-acoplados ${ }^{141,142}$, ou ainda na condução através de fios moleculares ${ }^{131,132,134,137,143}$.

Portanto, os oligômeros polimetálicos de valência mista também exercem um papel de fundamental importância neste campo.

Paralelamente aos estudos de transferência de elétrons óptica, um outro assunto de fundamental importância no desenvolvimento da eletrônica molecular corresponde aos processos fotoquímicos. Processos de transferência eletrônica fotoquimicamente induzidos são diferentes do análogo óptico porque ocorrem em duas etapas: a absorção inicial de um fóton por um cromóforo produz um estado eletronicamente excitado, que pode ser seguida de uma transferência de elétron(s) para outro grupo que suprime o estado excitado inici$\mathrm{al}^{27,71}$. Visto que o cromóforo e o supressor estão diretamente ligados por um ligante-ponte, a transferência do(s) elétron(s) entre eles é dependente das propriedades da ponte exatamente da mesma maneira que os exemplos de reações de transferência de elétrons óptica dados anteriormente.

Alternativamente, transferência de energia pode ocorrer no caso da absorção de luz por um cromóforo ser seguida pela emissão de luz de um outro, possibilitando, assim, a construção de fios moleculares fotônicos ${ }^{40,144}$. Exemplos interessantes de transferência de energia entre unidades metálicas conectadas por cadeias longas têm sido reportados ${ }^{39,40,145}$. A comunicação a longa distância entre cromóforo e supressor também depende das interações entre os centros, propagando-se através das redes de ligações. Portanto, esse tipo de interação também está sujeito a algum tipo de controle.

\section{Perspectivas}

Durante algum tempo (principalmente na década de 80), a importância dos compostos de valência mista esteve ligada à busca por materiais semicondutores e supercondutores. Os tão celebrados óxidos cerâmicos supercondutores em altas temperaturas são, na verdade, materiais de valência mista. Além disso, esse fenômeno foi crucial para a compreensão de muitos processos biológicos, incluindo os mecanismos de transferência eletrônica em metaloenzimas com múltiplos centros e da ligação de oxigênio molecular a enzimas.

Nos anos 90, a comunidade científica continuou demonstrando um interesse crescente nos compostos de valência mista e, com o surgimento de novas facilidades (métodos e técnicas modernas de investigação), este interesse deverá aumentar progressivamente neste século. Isso porque a presença de valência mista, em alguns tipos de sistemas, pode levar ao estabelecimento de um código binário de comutação, cuja manipulação desempenha um importante papel na procura de materiais eletrônicos e/ou opto-eletrônicos moleculares. O mesmo é válido para os dispositivos de armazenamento de energia.

Atualmente, os campos de pesquisa de compostos de valência mista envolvem as disciplinas de química, física e biologia. A ênfase teórica envolve desde dímeros simples a oligômeros mais complicados e até sólidos estendidos. Sínteses laboriosas estão concentradas no controle fino da transição de comportamento localizado/ deslocalizado. Técnicas espectroscópicas ópticas, magnéticas e de nêutrons têm sido particularmente bem-sucedidas no estudo de sistemas de valência mista, as quais permitiram o mapeamento e a modulação de estados vibracionais e magnéticos de baixa energia, bem como a exploração de características especiais de novos estados excitados, pelo emprego de espectroscopia e fotoeletroquímica ultrarápidas.

O impacto do controle sobre os processos de transferência de elétrons (bem como sobre os magneto-ópticos) estende-se desde as áreas tradicionais de eletrônica do estado sólido, transporte de elétrons em semicondutores e estudo de metaloenzimas com múltiplos centros redox até a mimetização de sistemas biológicos (p. ex. fotossíntese artificial), dispositivos de armazenamento de energia, sensores moleculares, e a área recente de eletrônica molecular e nanotecnologia.

\section{APÊNDICE}

\section{A base do formalismo de Hush}

O modelo de Hush ${ }^{5,59,60}$ para acoplamento eletrônico metal-metal, que é baseado em parametrização espectroscópica, teve a sua origem no tratamento perturbacional de Murrell ${ }^{146} \mathrm{e}$ foi derivado da teoria das interações doador-aceitador de Mulliken ${ }^{101}$.

De acordo com esse formalismo, o campo de potencial do íon aceitador não-reduzido é tratado como um termo de perturbação no Hamiltoniano que opera sobre a função de onda de ordem zero (não perturbada), $\varphi_{\mathrm{D}}$, que descreve o sistema quando o elétron ímpar a ser transferido se encontra completamente localizado sobre o sítio doador. $\mathrm{O}$ estado de ordem zero com o elétron totalmente localizado sobre o íon aceitador é denominado $\varphi_{\mathrm{A}}$.

As funções de onda resultantes (perturbadas) para ambos os estados fundamental (Eq. 41) e excitado (Eq. 42) são escritas como:

$\varphi_{\mathrm{D}}^{\prime}=\varphi_{\mathrm{D}}+\alpha \varphi_{\mathrm{A}}$

$\varphi_{\mathrm{A}}^{\prime}=\varphi_{\mathrm{A}}+\lambda \varphi_{\mathrm{D}}$

Murrell derivou as expressões perturbacionais para $\alpha$ e $\lambda$ para resultar as Eqs. 43 e 44, respectivamente.

$$
\begin{gathered}
\alpha=\frac{H_{\mathrm{AD}}-S_{\mathrm{AD}} H_{\mathrm{DD}}}{E_{\mathrm{D}}-E_{\mathrm{A}}} \\
\lambda=\frac{H_{\mathrm{AD}}-S_{\mathrm{AD}} H_{\mathrm{AA}}}{E_{\mathrm{A}}-E_{\mathrm{D}}}
\end{gathered}
$$

onde 


$$
H_{\mathrm{AD}}=\left\langle\varphi_{\mathrm{A}}|H| \varphi_{\mathrm{D}}\right\rangle
$$

e

$$
S_{\mathrm{AD}}=\left\langle\varphi_{\mathrm{A}} \mid \varphi_{\mathrm{D}}\right\rangle
$$

Assumindo-se que $|\lambda|>|\alpha|$, então o momento de transição (na aproximação do dipolo elétrico) entre $\varphi_{\mathrm{D}}$ ' e $\varphi_{\mathrm{A}}$ ' será dado pelas Eqs. 47 e 48.

$$
\begin{aligned}
& M_{\mathrm{D}^{\prime} \mathrm{A}^{\prime}}=\left\langle\varphi_{\mathrm{D}}^{\prime}|\vec{\mu}| \varphi_{\mathrm{A}}^{\prime}\right\rangle \\
& M_{\mathrm{D}^{\prime} \mathrm{A}^{\prime}}=\alpha\left(M_{\mathrm{AA}}=M_{\mathrm{DD}}\right)+\left(M_{\mathrm{DA}}-S_{\mathrm{AD}} M_{\mathrm{DD}}\right)
\end{aligned}
$$

Hush destacou que, no limite onde $S_{\mathrm{AD}} \sim 0$ (isto é, quando a integral de recobrimento entre $\varphi_{\mathrm{A}}$ e $\varphi_{\mathrm{D}}$ for negligenciável), a Eq. 48 se reduz à seguinte fórmula simplificada:

$M_{\mathrm{D}^{\prime} \mathrm{A}^{\prime}} \cong e_{0} \alpha r$

onde $e_{0}$ é a carga do elétron e $r$ (igual a $d$ na notação utilizada neste artigo) é a distância entre os sítios doador e aceitador.

A magnitude da força do oscilador, $f_{\text {osc }}$, por sua vez, pode ser estimada experimentalmente dos dados espectroscópicos pela Eq. $50^{5,147,148}$ e teoricamente pela Eq. $51^{5,94,147}$ :

$$
\begin{aligned}
& f_{\text {osc }}=4,32 \times 10^{-9} \int \varepsilon \mathrm{d} v \cong 4,32 \times 10^{-9} \cdot \varepsilon_{\text {máx }} \cdot \Delta \bar{v}_{1 / 2} \\
& f_{\text {osc }}=1,09 \times 10^{-5}\left(M_{\mathrm{DA}} / e_{0}\right)^{2} \bar{v}
\end{aligned}
$$

onde, na Eq. 50, a absortividade molar ( $\varepsilon$ ) e a largura de meia-banda $\left(\Delta \bar{v}_{1 / 2}\right)$ são dados em mol ${ }^{-1} \mathrm{dm}^{3} \mathrm{~cm}^{-1}$ (ou cm $\left.\mathrm{cm}^{2} 10^{3} / \mathrm{mol}\right) \mathrm{e} \mathrm{cm}^{-1}$, respectivamente ${ }^{149}$; e, na Eq. 51, as unidades de $M_{\mathrm{DA}}$ são elétron ångström (e $\AA$ ).

Dessa forma, o momento de transição é dado por:

$$
\left|M_{\mathrm{DA}}\right|=0,02\left(\frac{\varepsilon \cdot \Delta \overline{\mathrm{v}}_{1 / 2}}{\bar{v}}\right)^{1 / 2}
$$

e segue que

$$
\alpha^{2}=4,24 \times 10^{-4}\left(\frac{\varepsilon_{\text {máx }} \cdot \Delta \bar{v}_{1 / 2}}{\bar{v} \cdot d^{2}}\right)
$$

onde a distância $d=r_{\mathrm{DA}}$ é dada em $\AA$, e $\bar{v}$ é a energia de transição $\left(E_{\mathrm{op}}\right)$ em $\mathrm{cm}^{-1}$, que comumente é obtida de $\bar{v}_{\text {máx }}{ }^{150}$

A partir das Eqs. 43 e 44 no limite $S=0$, conclui-se que a energia de ressonância resultante da interação eletrônica (por transferência de carga) em um sistema fracamente acoplado é dada pela expressão final (Eq. 55) da Eq. 54, que é exatamente a fórmula de Hush introduzida nas seções anteriores.

$$
\begin{aligned}
& H_{\mathrm{AD}}=\alpha\left(E_{\mathrm{A}}-E_{\mathrm{D}}\right) \cong \alpha \cdot \bar{v}_{\text {máx }} \\
& H_{\mathrm{AD}}=\frac{2,05 \times 10^{-2}}{d} \cdot \sqrt{\varepsilon_{\text {máx }} \cdot \bar{v}_{\text {máx }} \cdot \Delta \bar{v}_{1 / 2}}
\end{aligned}
$$

Neste artigo, utilizou-se o símbolo $H_{\text {ab }}$ para se referir à energia de acoplamento eletrônico. Essa é a notação mais comum entre os químicos inorgânicos, apesar de não ser difícil encontrar outras formas como $H_{\mathrm{DA}}$ ou $J$.

\section{AGRADECIMENTOS}

À Fundação de Amparo à Pesquisa do Estado de São Paulo (FAPESP) e ao Conselho Nacional de Desenvolvimento Científico e Tecnológico $(\mathrm{CNPq})$ pelo apoio financeiro.

\section{REFERÊNCIAS E NOTAS}

1. Brown, D. B., ed. Em Mixed-Valence Compounds: Theory and Applications in Chemistry, Physics, Geology, and Biology; Reidel: Dordrecht, 1980.

2. Goffer, Z.; Archaeological Chemistry, Wiley: New York, 1980

3. Robin, M. B.; Day, P.; Adv. Inorg. Chem. Rad. 1967, 10, 247.

4. Allen, G. C.; Hush, N. S.; Prog. Inorg. Chem. 1967, 8, 357.

5. Hush, N. S.; Prog. Inorg. Chem. 1967, 8, 391.

6. Creutz, C.; Prog. Inorg. Chem. 1983, 30, 1.

7. Crutchley, R. J.; Adv. Inorg. Chem. Rad. 1994, 41, 273.

8. Ward, M. D.; Chem. Soc. Rev. 1995, 24, 121.

9. Rocha, R. C.; Tese de Doutorado, Universidade de São Paulo, Brasil, 2000.

10. Woodward, J.; Philos. Trans. Roy. Soc. London 1724, 33, 15.

11. Toma, H. E.; J. Inorg. Nucl. Chem. 1976, 38, 431; Toma, H. E.; Oliveira, L. A. A.; Inorg. Chim. Acta 1979, 33, L143; Toma, H. E.; Lellis, F. T. P.; An. Acad. Bras. Cienc. 1987, 59, 165; Toma, H. E.; Carter, J. M.; Santos, P. S.; J. Chem. Res. Synop. 1987, 226; Toma, H. E.; Lellis, F. T. P.; Spectrosc. Lett. 1988, 21, 45.

12. Matsumoto, F. M.; Temperini, M. L. A.; Toma, H. E.; Electrochim. Acta 1994, 38, 385; Mortimer, R. J.; Chem. Soc. Rev. 1997, 26, 147.

13. Mattos, I. L. de; Gorton, L.; Quim. Nova 2001, 24, 200.

14. Creutz, C.; Taube, H.; J. Am. Chem. Soc. 1969, 91, 3988; Creutz, C.; Taube, H.; J. Am. Chem. Soc. 1973, 95, 1086.

15. Cowan, D. O.; Kaufman, F.; J. Am. Chem. Soc. 1970, 92, 219; Cowan, D. O.; Levanda, C.; Park, J.; Kaufman, F.; Acc. Chem. Res. 1973, 6, 1.

16. Haim, A.; Adv. Chem. Ser. 1997, 253, 239.

17. Moreira, I. D.; Franco, D. W.; Adv. Chem. Ser. 1997, 253, 255

18. Rocha, R. C.; Toma, H. E.; Inorg. Chim. Acta 2000, 310, 65.

19. Rocha, R. C.; Toma, H. E.; Can. J. Chem. 2001, 79, 145

20. Shepherd, R. E.; Chen, Y.; Zhang, S. S.; Lin, F. T.; Kortes, R. A.; Adv. Chem. Ser. 1997, 253, 367; Chatterjee, D.; Coord. Chem. Rev. 1998, 168, 273; Buslaeva, T. M.; Red'kina, S. N.; Rudnitskaya, O. V.; Russ. J. Coord. Chem. 1999, 25, 1.

21. Aquino, M. A. S.; Coord. Chem. Rev. 1998, 170, 141.

22. Rocha, R. C.; Araki, K.; Toma, H. E.; Inorg. Chim. Acta 1999, $285,197$.

23. Alexiou, A. D. P.; Dovidauskas, S.; Toma, H. E.; Quim. Nova 2000, 23, 785

24. Wu, Y.; Pfennig, B. W.; Sharp, S. L.; Ludwig, D. R.; Warren, C. J.; Vicenzi, E. P.; Bocarsly, A. B.; Coord. Chem. Rev. 1997, 159, 245; Garcia, C. G.; De Lima, J. F.; Iha, N. Y. M.; Coord. Chem. Rev. 2000, 196, 219; Chang, C. C.; Pfennig, B.; Bocarsly, A. B.; Coord. Chem. Rev. 2000, 208, 33.

25. Batista, A. A.; Porcu, O. M.; Nascimento, O. R.; Barbosa, V. M.; Oliva, G.; J. Coord. Chem. 1993, 30, 345; Laidlaw, W. M.; Denning, R. G.; J. Chem. Soc., Dalton Trans. 1994, 1987; Brittingham, K. A.; Schreiner, S.; Gallaher, T. N.; J. Chem. Educ. 1995, 72, 941; Queiroz, S. L.; Batista, A. A.; Quim. Nova 1996, 19, 651; Yeomans, B. D.; Humphrey, D. G.; Heath, G. A.; J. Chem. Soc., Dalton Trans. 1997, 4153; Whittall, I. R.; Cifuentes, M. P.; Humphrey, M. G.; Luther-Davies, B.; Samoc, M.; Houbrechts, S.; Persoons, A.; Heath, G. A.; Hockless, D. C. R.; J. Organomet. Chem. 1997, 549, 127; Cotton, F. A.; Inorg. Chem. 1998, 37, 5710; MacFarlane, K. S.; Thorburn, I. S.; Cyr, P. W.; Chau, D.; Rettig, S. J.; James, B. R.; Inorg. Chim. Acta 1998, 270, 130; Mashima, K.; Kaneda, Y.; Fukumoto, A.; Tanaka, M.; Tani, K.; Nakano, H.; Nakamura, A.; Inorg. Chim. Acta 1998, 270, 459; Azam, K. A.; Hursthouse, M. B.; Islam, M. R.; Kabir, S. E.; Malik, K. M. A.; Miah, R.; Sudbrake, C.; Vahrenkamp, H.; J. Chem. Soc., Dalton Trans. 1998, 1097; Dinelli, L. R.; Batista, A. A.; Wohnrath, K.; de Araujo, M. P.; Queiroz, S. L.; Bonfadini, M. R.; Oliva, G.; Nascimento, O. R.; Cyr, P. W.; MacFarlane, K. S.; James, B. R.; Inorg. Chem. 1999, 38, 5341; Wohnrath, K.; de Araujo, M. P.; Dinelli, L. R.; Batista, A. A.; Moreira, I. D.; Castellano, E. E.; Ellena, J.; J. Chem. Soc., Dalton Trans. 2000, 3383.

26. Juris, A.; Balzani, V.; Barigelletti, F.; Belser, P.; von Zelewsky, A.; Coord. Chem. Rev. 1988, 84, 85; Balzani, V.; Juris, A.; Venturi, M.; Campagna, S.; Serroni, S.; Chem. Rev. 1996, 96, 759.

27. Sauvage, J.-P.; Collin, J.-P.; Chambron, J.-C.; Guillerez, S.; Coudret, C.; Balzani, V.; Barigelletti, F.; De Cola, L.; Flamigni, L.; Chem. Rev. 1994, 94, 993 
28. von Zelewsky, A.; Belser, P.; Hayoz, P.; Dux, R.; Hua, X.; Suckling, A. Stoecklievans, H.; Coord. Chem. Rev. 1994, 132, 75; Belser, P.; Bernhard, S.; Jandrasics, E.; von Zelewsky, A.; De Cola, L.; Balzani, V.; Coord. Chem. Rev. 1997, 159, 1; Keene, F. R.; Coord. Chem. Rev. 1997, 166, 121 Mongey, K. F.; Vos, J. G.; MacCraith, B. D.; McDonagh, C. M.; Coord. Chem. Rev. 1999, 186, 417.

29. Elliott, C. M.; Derr, D. L.; Ferrere, S.; Newton, M. D.; Liu, Y. P.; J. Am. Chem. Soc. 1996, 118, 5221.

30. Ward, M. D.; White, C. M.; Barigelletti, F.; Armaroli, N.; Calogero, G.; Flamigni, L.; Coord. Chem. Rev. 1998, 171, 481; De Cola, L.; Belser, P.; Coord. Chem. Rev. 1998, 177, 301

31. Joulié, L. F.; Schatz, E.; Ward, M. D.; Weber, F.; Yellowlees, L. J.; J. Chem. Soc., Dalton Trans. 1994, 799.

32. Whittle, B.; Everest, N. S.; Howard, C.; Ward, M. D.; Inorg. Chem. 1995, 34, 2025; Ward, M. D.; Inorg. Chem. 1996, 35, 1712; Keyes, T. E.; Forster, R. J.; Jayaweera, P. M.; Coates, C. G.; McGarvey, J. J.; Vos, J. G.; Inorg. Chem. 1998, 37, 5925; Shukla, A. D.; Whittle, B.; Bajaj, H. C.; Das, A.; Ward, M. D.; Inorg. Chim. Acta 1999, 285, 89.

33. Sedney, D.; Ludi, A.; Inorg. Chim. Acta 1981, 47, 153; Chou, M. H.; Creutz, C.; Sutin, N.; Inorg. Chem. 1992, 31, 2318; Constantino, V. R. L.; de Oliveira, L. F. C.; Santos, P. S.; Toma, H. E.; Transition Met. Chem. 1994, 19, 103; Rawson, J. M.; Winpenny, R. E. P.; Coord. Chem. Rev. 1995 139, 313; Das, N. N.; Das, R.; Polyhedron 1997, 16, 2135; Rein, F. N.; Toma, H. E.; Polyhedron 1998, 17, 1439.

34. Isied, S. S.; Adv. Chem. Ser. 1991, 229; Isied, S. S.; Adv. Chem. Ser. 1997, 331.

35. Moreira, I. D.; Franco, D. W.; Inorg. Chem. 1994, 33, 1607; Matsumoto, K.; Matsumoto, T.; Kawano, M.; Ohnuki, H.; Shichi, Y.; Nishide, T.; Sato, T.; J. Am. Chem. Soc. 1996, 118, 3597; Moreira, I. D.; Lima, E. C.; Franco, D. W.; Inorg. Chim. Acta 1998, 267, 93; Moreira, I. D.; Parente, L. T. D.; Franco, D. W.; Quim. Nova 1998, 21, 545; Samanta, R.; Munshi, P.; Santra, B. K.; Lahiri, G. K.; Polyhedron 1999, 18, 995.

36. Moreira, I. D.; de Lima, J. B.; Franco, D. W.; Coord. Chem. Rev. 2000 , 196, 197

37. Toma, H. E.; Araki, K.; Coord. Chem. Rev. 2000, 196, 307.

38. Seddon, E. A.; Seddon, K. R. Em The Chemistry of Ruthenium; Clark, R. J. H., ed.; Elsevier Science Pub.: Amsterdam, 1984

39. Frank, M.; Nieger, M.; Vogtle, F.; Belser, P.; von Zelewsky, A.; De Cola, L.; Balzani, V.; Barigelletti, F.; Flamigni, L.; Inorg. Chim. Acta 1996, 242, 281.

40. Collin, J.-P.; Gavina, P.; Heitz, V.; Sauvage, J.-P.; Eur. J. Inorg. Chem. 1998, 1.

41. Demadis, K. D.; Neyhart, G. A.; Kober, E. M.; Meyer, T. J.; J. Am. Chem. Soc. 1998, 120, 7121; Demadis, K. D.; Neyhart, G. A.; Kober, E. M.; White P. S.; Meyer, T. J.; Inorg. Chem. 1999, 38, 5948; Demadis, K. D.; ElSamanody, E. S.; Coia, G. M.; Meyer, T. J.; J. Am. Chem. Soc. 1999, 121 535; Demadis, K. D.; Neyhart, G. A.; Kober, E. M.; White, P. S.; Meyer T. J.; Inorg. Chem. 2000, 39, 3430.

42. Toma, H. E.; Santos, P. S.; Can. J. Chem. 1977, 55, 3549; Toma, H. E.; J. Coord. Chem. 1978, 7, 231.

43. Taube, H.; Adv. Chem. Ser. 1997, 253, 1.

44. Toma, H. E.; An. Acad. Bras. Cienc. 2000, 72, 1.

45. Barbara, P. F.; Meyer, T. J.; Ratner, M. A.; J. Phys. Chem. 1996, 100, 13148

46. Young, R. C.; Keene, F. R.; Meyer, T. J.; J. Am. Chem. Soc. 1977, 99, 2468.

47. Bazhenova, T. A.; Gruzelle, M.; Leny, G.; Shilov, A. E.; Shilova, A. K.; Kinet. Catal. 1981, 22, 1158; Bazhenova, T. A.; Lobkovskaya, R. M.; Shibaeva, R. P.; Shilov, A. E.; Shilova, A. K.; Gruzelle, M.; Leny, G.; Tchoubar, B.; J. Organomet. Chem. 1983, 244, 265.

48. Henry Taube, 1915 (Stanford University, CA, USA). Laureado com o Prêmio Nobel de Química de 1983 por sua contribuição em mecanismos de reações de transferência de elétrons, especialmente em complexos metálicos.

49. Taube, H.; Myers, H.; Rich, R. L.; J. Am. Chem. Soc. 1953, 75, 4118; Taube, H.; Myers, H.; J. Am. Chem. Soc. 1954, 76, 2103.

50. Meyer, T. J.; Taube, H. Em Comprehensive Coordination Chemistry Wilkinson, G.; Gillard, R. D.; McCleverty, J. A., eds.; Pergamon Press: Oxford, 1987, vol. 1, p. 331.

51. Gordon, B. N.; Williams, L. L.; Sutin, N.; J. Am. Chem. Soc. 1961, 83, 2061.

52. Fleischer, E. B.; Lavallee, D. K.; J. Am. Chem. Soc. 1972, 94, 2599.

53. Tom, G. M.; Creutz, C.; Taube, H.; J. Am. Chem. Soc. 1974, 96, 7828.

54. Sutton, J. E.; Sutton, P. M.; Taube, H.; Inorg. Chem. 1979, 18, 1017.

55. Sutton, J. E.; Taube, H.; Inorg. Chem. 1981, 20, 3125.

56. Rudolf A. Marcus, 1923 (California Institute of Technology, CA, USA); Laureado com o Prêmio Nobel de Química de 1992 por sua contribuição à teoria de reações de transferência de elétrons em sistemas químicos.

57. Marcus, R. A.; Discuss. Faraday Soc. 1960, 21; Marcus, R. A.; Annu. Rev. Phys. Chem. 1964, 15, 155
58. Marcus, R. A.; Angew. Chem., Int. Ed. Engl. 1993, 32, 1111; Marcus, R. A.; Adv. Chem. Phys. 1999, 106, 1.

59. Hush, N. S.; Trans. Faraday. Soc. 1961, 57, 557; Hush, N. S.; Electrochim. Acta 1968, 13, 1005.

60. Hush, N. S.; Coord. Chem. Rev. 1985, 64, 135

61. Dogonadze, R. R.; Levich, V. G.; Dokl. Akad. Nauk USSR 1959, 124, 123 ; Dogonadze, R. R.; Dokl. Akad. Nauk USSR 1960, 1108; Dogonadze, R. R.; Dokl. Akad. Nauk USSR 1960, 1368; Dogonadze, R. R.; Levich, V. G.; Dokl. Akad. Nauk USSR 1960, 133, 158; Levich, V. G.; Adv. Electrochem. Sci. Eng. 1966, 4, 249.

62. Marcus, R. A.; J. Electroanal. Chem. 2000, 483, 2.

63. A diferença de energia livre padrão de Gibbs, $\Delta G^{\mathrm{o}}$, para uma reação de transferência de elétrons é expressa pela equação de Rehm-Weller, onde os termos mais importantes são relacionados ao potencial de ionização do doador $\left(E_{\mathrm{ox}}{ }^{\mathrm{D}}\right)$, a afinidade eletrônica do aceitador $\left(E_{\text {red }}{ }^{\mathrm{A}}\right)$ e o fator eletrostático em função do solvente (função trabalho envolvida na aproximação das espécies reagentes ${ }^{64}$.

64. Rehm, D.; Weller, A.; Isr. J. Chem. 1970, 8, 259; Julliard, M.; Chanon, M.; Chem. Rev. 1983, 83, 425 .

65. Taube, H.; Adv. Inorg. Chem. Radiochem. 1959, 1, 1; Taube, H.; Adv. Chem. Ser. 1965, 14, 107; Taube, H.; J. Chem. Educ. 1968, 45, 452; Taube, H. Gould, E. S.; Acc. Chem. Res. 1969, 2, 321; Taube, H.; Pure Appl. Chem. 1970, 24, 289; Taube, H. Em Tunneling in Biological Systems; Chance B.; Devault, D. C.; Frauenfelder, H.; Marcus, R. A.; Schrieffer, J. R.; Sutin, N., eds.; Academic Press: New York, 1979; p 173; Taube, H.; Comment. Inorg. Chem. 1981, 1, 17; Taube, H.; Angew. Chem., Int. Ed. 1984, 23, 329; Taube, H.; Science 1984, 226, 1028.

66. Taube, H.; Electron Transfer Reactions of Complex Ions in Solution, Academic Press: New York, 1970.

67. Sutin, N.; Annu. Rev. Phys. Chem. 1966, 17, 119; Sutin, N.; Acc. Chem. Res. 1968, 1, 225; Sutin, N. Em Inorganic Biochemistry; Eichhorn, G. L. Ed. Elsevier; New York, 1973; Vol. 2, p 611; Sutin, N.; Acc. Chem. Res. 1982, 15, 275; Sutin, N.; Prog. Inorg. Chem. 1983, 30, 441; Sutin, N.; Brunschwig, B. S.; Adv. Chem. Ser. 1990, 65; Sutin, N.; Adv. Chem. Ser. 1991, 25.

68. Newton, M. D.; Sutin, N.; Annu. Rev. Phys. Chem. 1984, 35, 437

69. Marcus, R. A.; Sutin, N.; Comments Inorg. Chem. 1986, 5, 119.

70. Haim, A.; Acc. Chem. Res. 1975, 8, 264; Haim, A.; Prog. Inorg. Chem 1983, 30, 273; Haim, A.; Pure Appl. Chem. 1983, 55, 89.

71. Turro, N. J.; Modern Molecular Photochemistry, Benjamin/Cummings: California, 1978; Balzani, V.; Scandola, F.; Supramolecular Photochemistry Ellis-Horwood: Chichester, 1991.

72. Sutin, N.; Creutz, C.; Pure Appl. Chem. 1980, 52, 2717; Meyer, T. J.; Prog. Inorg. Chem. 1983, 30, 389; Kavarnos, G. J.; Turro, N. J.; Chem. Rev. 1986 86, 401; Balzani, V., ed.; Supramolecular Photochemistry; Reidel; Dordrecht, 1987; Billing, R.; Coord. Chem. Rev. 1997, 159, 257; De Silva A. P.; Fox, D. B.; Huxley, A. J. M.; McClenaghan, N. D.; Roiron, J.; Coord. Chem. Rev. 1999, 186, 297; Flamigni, L.; Barigelletti, F.; Armaroli, N. Collin, J.-P.; Dixon, I. M.; Sauvage, J.-P.; Williams, J. A. G.; Coord. Chem. Rev. 1999, 192, 671.

73. Fox, M. A.; Chanon, M., eds.; Photoinduced Electron Transfer, Elsevier: Amsterdam, 1988; Kavarnos, G. J.; Fundamentals of Photoinduced Electron Transfer, VCH: New York, 1993.

74. Willner, I.; Kaganer, E.; Joselevich, E.; Durr, H.; David, E.; Gunter, M. J.; Johnston, M. R.; Coord. Chem. Rev. 1998, 171, 261.

75. Reações de transferência de elétrons são usual e convenientemente representadas por meio de curvas parabólicas de variação de energia potencial (sistemas discretos e/ou em fase gasosa) ou energia livre (maioria dos sistemas complexos em fase condensada) ao longo da reação em função das coordenadas nucleares dos sistemas (posições, orientações, distâncias e ângulos de ligação, etc) ${ }^{57,62,76}$. Essa representação bidimensional simplificada é, na verdade, o perfil da interseção entre as duas superfícies multidimensionais de energia dos estados reagente e produto na direção da reação. As duas curvas se cruzam em um ponto de não-equilíbrio, no qual ocorre a transferência de elétrons térmica quando o elétron move-se do doador para o aceitador (isto é, ocorre a passagem do reagente ao produto).

76. Lewis, N. A.; J. Chem. Educ. 1980, 57, 478.

77. Ulstrup, J.; Jortner, J.; Chem. Phys. 1975, 63, 4358; Jortner, J.; J. Chem. Phys. 1976, 64, 4860; Hopfield, J. J.; Biophys. J. 1977, 18, 311; Beratan, D. N.; Onuchic, J. N.; Adv. Chem. Ser. 1991, 71; Beratan, D. N.; Betts, J. N.; Onuchic, J. N.; Science 1991, 252, 1285; Gehlen, J. N.; Daizadeh, I.; Stuchebrukhov, A. A.; Marcus, R. A.; Inorg. Chim. Acta 1996, 243, 271; Balabin, I. A.; Onuchic, J. N.; J. Phys. Chem. 1996, 100, 11573; LopezCastillo, J.-M.; Jay-Gerin, J.-P.; J. Phys. Chem. 1996, 100, 14289; Newton, M. D.; J. Electroanal. Chem. 1997, 438, 3; Kharkats, Y. I.; Ulstrup, J.; Chem. Phys. Lett. 1999, 303, 320. 
78. Chance, B.; Devault, D. C.; Frauenfelder, H.; Marcus, R. A.; Schrieffer, J. R.; Sutin, N., eds.; Tunneling in Biological Systems, Academic Press: New York, 1979.

79. Arnaut, L. G.; Formosinho, S. J.; J. Photochem. Photobiol., A 1997, 111, 111.

80. Wherland, S.; Gray, H. B.; Proc. Natl. Acad. Sci. U.S.A. 1976, 73, 2950; Addison, A. W.; Cullen, W. R.; Dolphin, D.; James, B. R., eds.; Biological Aspects of Inorganic Chemistry, Wiley: New York, 1977; Isied, S. S.; Prog. Inorg. Chem. 1984, 32, 443; Isied, S. S.; Adv. Chem. Ser. 1990, 226, 91; Therien, M. J.; Bowler, B. E.; Selman, M. A.; Gray, H. B.; Chang, I. J.; Winkler, J. R.; Adv. Chem. Ser. 1991, 191; Therien, M. J.; Chang, J.; Raphael, A. L.; Bowler, B. E.; Gray, H. B.; Struct. Bond. 1991, 75, 109; Lloyd, E.; Chapman, K.; Chapman, S. K.; Jia, Z. S.; Lim, M. C.; Tomkinson, N. P.; Salmon, G. A.; Sykes, A. G.; J. Chem. Soc., Dalton Trans. 1994, 675; Scott, J. R.; Fairris, J. L.; McLean, M.; Wang, K. F.; Sligar, S. G.; Durham, B.; Millett, F.; Inorg. Chim. Acta 1996, 243, 193; Harris, M. R.; Davis, D. J.; Durham, B.; Millett, F.; Biochim. Biophys. Acta 1997, 1319, 147; Gray, H. B.; Winkler, J. R.; J. Electroanal. Chem. 1997, 438, 43; Sun, J.; Wishart, J. F.; Inorg. Chem. 1998, 37, 1124; Wilker, J. J.; Dmochowski, I. J.; Dawson, J. H.; Winkler, J. R.; Gray, H. B.; Angew. Chem., Int. Ed. 1999, 38, 90; Malmstrom, B. G.; Wittung-Stafshede, P.; Coord. Chem. Rev. 1999, 186, 127.

81. Gray, H. B.; Winkler, J. R.; Аnnu. Rev. Biochem. 1996, 65, 537.

82. Turro, N. J.; Barton, J. K.; Tomalia, D. A.; Acc. Chem. Res. 1991, 24, 332; Turro, C.; Evenzahav, A.; Bossmann, S. H.; Barton, J. K.; Turro, N. J.; Inorg. Chim. Acta 1996, 243, 101; Arkin, M. R.; Stemp, E. D. A.; Turro, C.; Turro, N. J.; Barton, J. K.; J. Am. Chem. Soc. 1996, 118, 2267; KirschDe Mesmaeker, A.; Lecomte, J.-P.; Kelly, J.-M.; Top. Curr. Chem. 1996, 177, 25; Clarke, M. J.; Adv. Chem. Ser. 1997, 349; Stemp, E. D. A.; Arkin, M. R.; Barton, J. K.; J. Am. Chem. Soc. 1997, 119, 2921; Hall, D. B.; Barton, J. K.; J. Am. Chem. Soc. 1997, 119, 5045; Netzel, T. L.; J. Chem. Educ. 1997, 74, 646; Ortmans, I.; Moucheron, C.; Kirsch-De Mesmaeker, A.; Coord. Chem. Rev. 1998, 168, 233; Xiong, Y.; Ji, L. N.; Coord. Chem. Rev. 1999, 186, 711; Stemp, E. D. A.; Holmlin, R. E.; Barton, J. K.; Inorg. Chim. Acta 2000, 297, 88.

83. Homlin, R. E.; Danliker, P. J.; Barton, J. K.; Angew. Chem., Int. Ed. 1995, 34, 352; Meade, T. J.; Met. Ions Biol. Syst. 1996, 32, 453; Stemp, E. D. A.; Barton, J. K.; Met. Ions Biol. Syst. 1996, 33, 325; Barton, J. K.; Pure Appl. Chem. 1998, 70, 873; Giese, B.; Acc. Chem. Res. 2000, 33, 631.

84. Ulstrup, J.; Charge Transfer Processes in Condensed Media, SpringerVerlag: Berlin, 1979

85. Cannon, R. D.; Electron Transfer Reactions, Butterworths: London, 1980; Zuckerman, J. J., ed.; Inorganic Reactions and Methods, John Wiley \& Sons: New York, 1986, vol. 15; Eberson, L.; Electron Transfer Reactions in Organic Chemistry, Berlin, 1987; Gratzel, M.; Heterogeneous Photochemical Electron Transfer, CRC Press, 1989; Muller, A.; Ratajczak, H.; Junge, W.; Diemann, E., eds.; Electron and Proton Transfer in Chemistry and Biology, Elsevier: New York, 1992; Astruc, D.; Electron Transfer and Radical Processes in Transition Metal Chemistry, VCH: New York, 1995; Dwayne Miller, R. J.; McLendon, G.; Nozik, A. J.; Schmickler, A.; Willig, F.; Surface Electron Transfer Processes, VCH Pub.: New York, 1995; Isied, S. S., ed.; Electron Transfer Reactions: Inorganic, Organometallic, and Biological Applications, American Chemical Society: Washington, DC, 1997; Kuznetsov, A. M.; Ulstrup, J.; Electron Transfer in Chemistry and Biology: An Introduction to the Theory, John Wiley \& Sons: New York, 1998; Jortner, J.; Bixon, M., eds.; Electron Transfer - From Isolated Molecules to Biomolecules, John Wiley: New York, 1999.

86. Mattay, J., ed.; Photoinduced Electron Transfer, Springer-Verlag:; Berlin, 1989-1993 (vários volumes); Mariano, P. S., ed.; Advances in Electron Transfer Chemistry, JAI Press: London, 1991-1999 (vários volumes); Zuckerman, J. J., ed.; Inorganic Reactions and Methods, VCH Pub.: New York, 1994; Balzani, V.; Piotrowiak, P.; Rodgers, M. A. J., eds.; Electron Transfer in Chemistry, Wiley-VCH: Weinheim, 2001.

87. Toma, H. E.; J. Chem. Soc., Dalton Trans. 1980, 471

88. Piepho, S. B.; Krausz, E. R.; Schatz, P. N.; J. Am. Chem. Soc. 1978, 100, 2996.

89. Piepho, S. B.; Krausz, E. R.; Schatz, P. N.; J. Am. Chem. Soc. 1978, 100, 6319; Wong, K. Y.; Schatz, P. N.; Piepho, S. B.; J. Am. Chem. Soc. 1979, 101, 2793; Schatz, P. N. Em Mixed-Valence Compounds, Brown, D. B., ed.; Proceedings of the NATO Advanced Study Institute (58), Reidel: Dordrecht, 1980, p. 115; Piepho, S. B.; Nouv. J. Chim. 1980, 4, 639; Wong, K. Y.; Schatz, P. N.; Prog. Inorg. Chem. 1981, 28, 369; Piepho, S. B.; J. Am. Chem. Soc. 1990, 112, 4197; Reimers, J. R.; Hush, N. S.; Chem. Phys. 1996, 208, 177; Boukheddaden, K.; Varret, F.; Chem. Phys. 1997, 216, 373; Rezvani, A. R.; Bensimon, C.; Cromp, B.; Reber, C.; Greedan, J. E.; Kondratiev, V. V.; Crutchley, R. J.; Inorg. Chem. 1997, 36, 3322; BorrasAlmenar, J. J.; Coronado, E.; Ostrovsky, S. M.; Palii, A. V.; Tsukerblat, B. S.; Chem. Phys. 1999, 240, 149.
90. Apesar de vários modelos com ênfase nas propriedades de esfera interna terem sido descritos ${ }^{11}$, o tratamento vibrônico de Piepho, Krausz e Schatz (que é conhecido por modelo PKS) ${ }^{88}$ tem recebido maior atenção. Embora esse formalismo seja quanticamente mais rigoroso, apenas os modos vibracionais internos e simétricos são levados em conta, por meio de freqüências médias concernentes às ligações metal-ligante. Isso tem limitado a sua aplicação a alguns poucos sistemas deslocalizados, já que em sistemas de perturbação moderada os modos do solvente (efeitos do meio) se tornam bastante relevantes. Nesse aspecto, o modelo clássico de Hush $^{5}$ apresenta vantagens. Além disso, o último é mais comumente utilizado pela pouca complexidade teórica e por sua parametrização espectroscópica, tornando mais viável a tarefa de se comparar dados com séries de sistemas reportados em literatura.

91. Mayoh, B.; Day, P.; J. Am. Chem. Soc. 1972, 94, 2885; Mayoh, B.; Day, P.; Inorg. Chem. 1974, 13, 2273; Ratner, M.; Ondrechen, M.; Mol. Phys. 1976, 32, 1233; Lauher, J.; Inorg. Chim. Acta 1980, 39, 119; Burdett, J. E.; Comment. Inorg. Chem. 1981, 1, 85; Zhang, L.-T.; Ko, J.; Ondrechen, M. J.; J. Phys. Chem. 1989, 93, 3030.

92. O fator de adiabaticidade $\kappa$ é o coeficiente de transmissão eletrônica que dá a probabilidade de um elétron passar do estado inicial do reagente para o estado final do produto no ponto de interseção de suas superfícies em uma reação térmica $(Q=0,0$; onde as configurações nucleares de ambos devem ser idênticas para que se processe a transferência eletrônica). Por sua vez, essa probabilidade dependerá do grau de mistura eletrônica entre os dois estados, que é representada pela energia de ressonância, $J=H_{\mathrm{RP}}=$ $H_{\text {ab. }}$. A constante de velocidade de transferência de elétrons dada pela equação de Eyring $\left(k=\kappa \times v_{\mathrm{n}} \times \exp \left(-\Delta G^{\ddagger} / \mathrm{R} T\right)\right)$ contém um termo préexponencial representado pelo produto do fator de freqüência nuclear, $v$ (cujo valor médio em solução é assumido como $6 \times 10^{12} \mathrm{~s}^{-1}$ ) e da constante de adiabaticidade definida acima. Assim, Marcus tem chamado de adiabáticas $(\kappa=1)$ as reações em que o desdobramento de $2 \times H_{\mathrm{ab}}$ em $Q=$ 0,0 é grande o suficiente para não limitar a velocidade de transferência eletrônica. Quanto menor for $H_{\mathrm{ab}}$, menos adiabática e, conseqüentemente, mais lenta se tornará a reação. Quando $0 \leq \kappa<1$, então a reação é nãoadiabática. Newton e Sutin ${ }^{68}$ propuseram o valor de $H_{\mathrm{ab}}=0,025 \mathrm{eV}$ como critério de distinção entre transferência de elétrons adiabática e nãoadiabática. Em uma situação limite, a estabilização por ressonância é tão grande que apenas um mínimo de energia correspondendo ao estado fundamental do sistema (deslocalizado) é observado. A teoria de Marcus só se aplica corretamente para as reações de transferência de elétrons adiabáticas, que é o caso mais freqüente.

93. Jortner, J.; Pullman, B., eds.; Tunneling, Reidel: Dordrecht, 1986; Compton, R. G., ed.; Electron Tunneling in Chemistry, Elsevier Science Pub.: New York, 1989.

94. Szabo, A.; Ostlund, N. S.; Modern Quantum Chemistry: Introduction to Advanced Electronic Structure Theory, McGraw-Hill: New York, 1989; Levine, I. N.; Quantum Chemistry, $4^{\mathrm{a}}$ ed., Prentice-Hall: Englewood Cliffs, 1991.

95. Kaim, W.; Kasack, V.; Inorg. Chem. 1990, 29, 4696.

96. Richardson, D. E.; Taube, H.; Inorg. Chem. 1981, 20, 1278; Richardson, D. E.; Taube, H.; Coord. Chem. Rev. 1984, 60, 107.

97. Estudos com ligantes saturados e/ou espiralados de várias extensões têm demonstrado que hiperconjugação- $\sigma$ também pode promover uma ressonância doador-aceitador bastante significativa, cuja magnitude não pode ser justificada exclusivamente com base em efeitos de tunelamento através da rede de ligações do tipo $\sigma^{6,8}$.

98. Uma possível complicação é a existência de múltiplas transições de intervalência no espectro de baixa energia em alguns sistemas particulares. Baixa simetria molecular e acoplamento spin-órbita desdobram os orbitais $d \pi$, provocando transições sobrepostas de cada um dos orbitais cheios do íon $\mathrm{M}^{\mathrm{II}}$ para o orbital semipreenchido do íon $\mathrm{M}^{\mathrm{III}}: d \pi_{1}^{2}, d \pi_{2}^{2}, d \pi_{3}^{2}$ ( $\left.\mathrm{M}^{\mathrm{II}}\right)$ $\rightarrow d \pi_{3}{ }^{1}\left(\mathrm{M}_{\mathrm{b}}{ }_{\mathrm{b}}^{\mathrm{II}}\right)$. Somente a transição de menor energia $d \pi_{3}{ }^{2}\left(\mathrm{M}_{\mathrm{a}}{ }^{\mathrm{II}}\right) \rightarrow d \pi_{3}{ }^{1}$ $\left(\mathrm{M}_{\mathrm{b}}^{\mathrm{II}}\right)$ é relevante para a transferência de elétron(s) térmica, desde que as outras possíveis transições geram estados excitados interconfiguracionais em $\mathrm{M}^{\mathrm{III}}$. Dessa forma, o processo $d \pi_{1}{ }^{2}\left(\mathrm{M}_{\mathrm{a}}^{\mathrm{II}}\right) \rightarrow d \pi_{3}{ }^{1}\left(\mathrm{M}_{\mathrm{b}} \mathrm{II}\right)$ deve resultar em M ${ }^{\mathrm{III}}$ a configuração $d \pi_{1}{ }^{2}, d \pi_{2}{ }^{2}, d \pi_{3}{ }^{1} \mathrm{em}$ vez de $d \pi_{1}{ }^{1}, d \pi_{2}{ }^{2}, d \pi_{3}{ }^{2} .99$

99. Kober, E. M.; Goldsby, K. A.; Narayana, D. N. S.; Meyer, T. J.; J. Am. Chem. Soc. 1983, 105, 4303.

100. Robert S. Mulliken, 1896-1986 (University of Chicago, IL, USA). Laureado com o Prêmio Nobel de Química de 1966 por seu trabalho fundamental sobre ligações químicas e estrutura eletrônica de moléculas pelo método dos orbitais moleculares.

101. Mulliken, R. S.; J. Am. Chem. Soc. 1950, 72, 600; Mulliken, R. S.; J. Am. Chem. Soc. 1952, 64, 811; Mulliken, R. S.; Person, W. B.; Molecular Complexes, Wiley: New York, 1969. 
102. O parâmetro $d$ no denominador da Eq. 29 se refere, na verdade, à distância de transferência eletrônica "real" (isto é, $\Delta \vec{\mu} / e$ ), e não à separação geométrica metal-metal. Estudos recentes de espectroscopia Stark ${ }^{103}$ têm demonstrado que $d_{\mathrm{ET}}$ é normalmente menor que a distância de separação entre os centros metálicos doador e aceitador, $d_{\mathrm{M}-\mathrm{M}}$. No entanto, valores de $d_{\mathrm{ET}}$ nem sempre são acessíveis, já que a sua determinação envolve o uso de técnicas não-convencionais e pouco disponíveis. Por isso é que a grande maioria dos dados reportados na literatura atualmente envolvem $d_{\mathrm{M}-\mathrm{M}} \mathrm{e}$ não $d_{\mathrm{ET}}$. Contudo, é importante reconhecer que as magnitudes da energia de acoplamento eletrônico e da deslocalização determinadas dessa forma são intrinsecamente subestimadas.

103. Karki, L.; Lu, H. P.; Hupp, J. T.; J. Phys. Chem. 1996, 100, 15637; Bublitz, G. U.; Boxer, S. G.; Annu. Rev. Phys. Chem. 1997, 48, 213; Bublitz, G. U.; Laidlaw, W. M.; Denning, R. G.; Boxer, S. G.; J. Am. Chem. Soc. 1998, 120, 6068; Vance, F. W.; Slone, R. V.; Stern, C. L.; Hupp, J. T.; Chem. Phys. 2000, 253, 313.

104. Conforme deve ter sido notado, os diagramas de energia em função da configuração nuclear foram representados por energia livre de Gibbs, enquanto muitos outros autores utilizam curvas de energia potencial. Por isso é importante comentar que, de acordo com Marcus e Sutin ${ }^{69}$, energias de transição óptica (tais como $E_{\mathrm{IT}}$ ) estão relacionadas à variação de energia livre, e não de entalpia ou de energia interna.

105. Uma diferença conceitual bastante importante entre transferência de elétrons óptica e térmica é que, no processo induzido por calor, deve haver um rearranjo estrutural que precede a etapa de transferência eletrônica propriamente dita. Isto é, o sistema deve atingir a configuração representada no ponto de interseção entre as curvas de energia do reagente e do produto, onde as coordenadas nucleares de ambos os estados são idênticas. Em decorrência do princípio de Franck-Condon ${ }^{106}$, no processo induzido por luz a transição entre os estados ocorre verticalmente, sem rearranjo nuclear precedendo a etapa de transferência eletrônica. Portanto, imediatamente após a transferência do(s) elétron(s), o produto se encontrará na configuração nuclear de equilíbrio do reagente. Posteriormente, o sistema vibracionalmente excitado relaxa (dentro da escala de tempo vibracional) para resultar o produto em seu estado fundamental de equilíbrio ${ }^{106}$.

106. O princípio de Franck-Condon ${ }^{94}$ implica que, durante uma etapa elementar de transferência de elétrons (térmica ou fotoinduzida), as posições dos átomos (esferas interna ou externa) permanecem inalteradas. Ou seja, como as frequiências de deslocamento vibracional $\left(10^{12}-10^{13} \mathrm{~s}^{-1}\right)$, orientação dos dipolos do solvente $\left(10^{11}-10^{12} \mathrm{~s}^{-1}\right)$ e difusional $\left(10^{9}-10^{11} \mathrm{~s}^{-1}\right)$ são muito menores que a eletrônica $\left(\sim 10^{15} \mathrm{~s}^{-1}\right)$, o processo de transferência eletrônica é praticamente instantâneo em relação às etapas de rearranjo nuclear que o precedem/sucedem ${ }^{107}$.

107. Libby, W. F.; J. Phys. Chem. 1952, 56, 863; Libby, W. F.; J. Chem. Phys. 1963, 38, 420 .

108. Equivale à energia de Franck-Condon em sistemas simétricos.

109. Cannon, R. D.; Chem. Phys. Lett. 1977, 49, 299.

110. Powers, M. J.; Meyer, T. J.; J. Am. Chem. Soc. 1980, 102, 1289.

111. Brunschwig, B. S.; Ehrenson, S.; Sutin, N.; J. Phys. Chem. 1986, 90, 3657; Brunschwig, B. S.; Ehrenson, S.; Sutin, N.; J. Phys. Chem. 1987, 91, 4714

112. Hupp, J. T.; Neyhart, G. A.; Meyer, T. J.; J. Am. Chem. Soc. 1986, 108, 5349; Neyhart, G. A.; Hupp, J. T.; Curtis, J. C.; Timpson, C. J.; Meyer, T. J.; J. Am. Chem. Soc. 1996, 118, 3724.

113. Toma, H. E.; Takasugi, M. S.; J. Solution Chem. 1983, 12, 547; Toma, H. E.; Takasugi, M. S.; J. Solution Chem. 1989, 18, 575; Toma, H. E.; Takasugi, M. S.; Polyhedron 1989, 8, 941; Timpson, C. J.; Bignozzi, C A.; Sullivan, B. P.; Kober, E. M.; Meyer, T. J.; J. Phys. Chem. 1996, 100, 2915.

114. Curtis, J. C.; Sullivan, B. P.; Meyer, T. J.; Inorg. Chem. 1983, 22, 224

115. Blackbourn, R. L.; Hupp, J. T.; J. Phys. Chem. 1988, 92, 2817.

116. Mayer, U.; Gutmann, V.; Gerger, W.; Monatsh. Chem. 1975, 106, 1235; Gutmann, V.; Electrochim. Acta 1976, 21, 661; Gutmann, V.; The DonorAcceptor Approach to Molecular Interactions, Plenum: New York, 1980; Gutmann, V.; Resch, G.; Linert, W.; Coord. Chem. Rev. 1982, 43, 133.

117. Stranks, D. R.; Discuss. Faraday Soc. 1960, 29, 73; Meyer, T. J.; Taube, H.; Inorg. Chem. 1968, 7, 2369; Endicott, J. F.; Kumar, K.; Ramasami, T.; Rotzinger, F. P.; Prog. Inorg. Chem. 1983, 30, 141; Cotton, F. A.; Wilkinson, G.; Advanced Inorganic Chemistry, $5^{\text {a }}$ ed., Wiley: New York, 1987.

118. Hupp, J. T.; Neyhart, G. A.; Meyer, T. J.; Kober, E. M.; J. Phys. Chem. 1992, 96, 10820.

119. Nelsen, S. F.; Adamus, J.; Wolff, J.; J. Am. Chem. Soc. 1994, 116, 1589; Elliott, C. M.; Derr, D. L.; Matyushov, D. V.; Newton, M. D.; J. Am. Chem. Soc. 1998, 120, 11714.

120. Dubicki, L.; Ferguson, J.; Krausz, E. R.; J. Am. Chem. Soc. 1985, 107, 179; Dubicki, L.; Ferguson, J.; Krausz, E. R.; Lay, P. A.; Maeder, M.; Magnuson, R. H.; Taube, H.; J. Am. Chem. Soc. 1985, 107, 2167.
121. Somente para o complexo de Creutz-Taube, por exemplo, existe mais de uma centena de artigos dedicados especificamente a esse problema.

122. Joachim, C.; Launay, J.-P.; Chem. Phys. 1986, 109, 93; Woitellier, S.; Joachim, C.; Launay, J.-P.; Chem. Phys. 1989, 131, 481; Reimers, J. R.; Hush, N. S.; Inorg. Chem. 1990, 29, 3686; Webb, R. J.; Hagen, P. M.; Wittebort, R. J.; Sorai, M.; Hendrickson, D. N.; Inorg. Chem. 1992, 31, 1791; Dong, T.-Y.; Huang, C.-H.; Chang, C.-K.; Wen, Y.-S.; Lee, S.-L.; Chen, J.-A.; Yeh, W.-Y.; Yeh, A.; J. Am. Chem. Soc. 1993, 115, 6357; Das, A.; Maher, J. P.; McCleverty, J. A.; Badiola, J. A.; Ward, M. D.; J. Chem. Soc., Dalton Trans. 1993, 681; Pierce, D. T.; Geiger, W. E.; Inorg. Chem. 1994, 33, 373; Ribou, A.-C.; Launay, J.-P.; Takahashi, K.; Nihira, T.; Tarutani, S.; Spangler, C. W.; Inorg. Chem. 1994, 33, 1325; Reimers, J. R.; Hush, N. S.; J. Phys. Chem. A 1999, 103, 3066.

123. Launay, J.-P.; Coudret, C.; Ann. N.Y. Acad. Sci. 1998, 852, 116.

124. Metcalfe, R. A.; Dodsworth, E. S.; Lever, A. B. P.; Pietro, W. J.; Stufkens, D. J.; Inorg. Chem. 1993, 32, 3581; Masui, H.; Freda, A. L.; Zerner, M. C.; Lever, A. B. P.; Inorg. Chem. 2000, 39, 141.

125. Lindoy, L. F.; The Chemistry of Macrocyclic Ligand Complexes; CUP, 1989; DelMedico, A.; Fielder, S. S.; Lever, A. B. P.; Pietro, W. J.; Inorg. Chem. 1995, 34, 1507; Laine, P.; Marvaud, V.; Gourdon, A.; Launay, J.P.; Argazzi, R.; Bignozzi, C. A.; Inorg. Chem. 1996, 35, 711; Archut, A.; Vogtle, F.; De Cola, L.; Azzellini, G. C.; Balzani, V.; Ramanujam, P. S.; Berg, R. H.; Chem.-Eur. J. 1998, 4, 699; Archut, A.; Azzellini, G. C.; Balzani, V.; De Cola, L.; Vogtle, F.; J. Am. Chem. Soc. 1998, 120, 12187; Vogtle, F.; Plevoets, M.; Nieger, M.; Azzellini, G. C.; Credi, A.; De Cola, L.; De Marchis, V.; Venturi, M.; Balzani, V.; J. Am. Chem. Soc. 1999, 121, 6290; Fraysse, S.; Coudret, C.; Launay, J.-P.; Eur. J. Inorg. Chem. 2000, 1581.

126. Neyhart, G. A.; Meyer, T. J.; Inorg. Chem. 1986, 25, 4808; Loeb, B. L.; Neyhart, G. A.; Worl, L. A.; Danielson, E.; Sullivan, B. P.; Meyer, T. J.; J. Phys. Chem. 1989, 93, 717; Launay, J.-P.; Tourrelpagis, M.; Lipskier, J. F.; Marvaud, V.; Joachim, C.; Inorg. Chem. 1991, 30, 1033; Haga, M.; Ano, T.; Kano, K.; Yamabe, S.; Inorg. Chem. 1991, 30, 3843; Marvaud, V.; Launay, J.-P.; Inorg. Chem. 1993, 32, 1376; Haga, M. A.; Ali, M. M.; Koseki, S.; Fujimoto, K.; Yoshimura, A.; Nozaki, K.; Ohno, T.; Nakajima, K.; Stufkens, D. J.; Inorg. Chem. 1996, 35, 3335; Chao, H.; Ye, B. H.; Zhang, Q. L.; Ji, L. N.; Inorg. Chem. Commun. 1999, 2, 338; Hossain, M. D.; Ueno, R.; Haga, M.; Inorg. Chem. Commun. 2000, 3, 35.

127. Rocha, R. C.; Toma, H. E.; Inorg. Chem. Commun. 2001, 4, 230.

128. Lowery, M. D.; Hammack, W. S.; Drickamer, H. G.; Hendrickson, D. N.; J. Am. Chem. Soc. 1987, 109, 8019; Lewis, N. A.; Obeng, Y. S.; J. Am. Chem. Soc. 1989, 111, 7624; Curtis, J. C.; Roberts, J. A.; Blackbourn, R. L.; Dong, Y. H.; Massum, M.; Johnson, C. S.; Hupp, J. T.; Inorg. Chem. 1991, 30, 3856; Roberts, J. A.; Hupp, J. T.; Inorg. Chem. 1992, 31, 157; Dong, Y.; Hupp, J. T.; Yoon, D. I.; J. Am. Chem. Soc. 1993, 115, 4379; Hupp, J. T.; Dong, Y.; Inorg. Chem. 1994, 33, 4421; Zhang, X. K.; Kankel, C. R.; Hupp, J. T.; Inorg. Chem. 1994, 33, 4738; Naklicki, M. L.; Crutchley, R. J.; Inorg. Chim. Acta 1994, 225, 123; Lau, K. W.; Hu, A. M.-H.; Yen, M. H.-J.; Fung, E. Y.; Grzybicki, S.; Matamoros, R.; Curtis, J. C.; Inorg. Chim. Acta 1994, 226, 137; Neyhart, G. A.; Timpson, C. J.; Bates, W. D.; Meyer, T. J.; J. Am. Chem. Soc. 1996, 118, 3730.

129. Debreczeny, M. P.; Svec, W. A.; Wasielewski, M. R.; Science 1996, 274, 584; Ward, M. D.; Chem. Ind. 1997, 640; Bergonzi, R.; Fabbrizzi, L.; Licchelli, M.; Mangano, C.; Coord. Chem. Rev. 1998, 170, 31.

130. Arrhenius, T. S.; Blancharddesce, M.; Dvolaitzky, M.; Lehn, J.-M.; Malthete, J.; Proc. Natl. Acad. Sci. U.S.A. 1986, 83, 5355; Gust, D.; Nature 1994, 372, 133; Ward, M. D.; Chem. Ind. 1996, 568; Paul, F.; Lapinte, C.; Coord. Chem. Rev. 1998, 180, 431; Constable, E. C.; Housecroft, C. E.; Schofield, E. R.; Encinas, S.; Armaroli, N.; Barigelletti, F.; Flamigni, L.; Figgemeier, E.; Vos, J. G.; Chem. Commun. 1999, 869; Anderson, H. L.; Chem. Commun. 1999, 2323; Barigelletti, F.; Flamigni, L.; Chem. Soc. Rev. 2000, 29, 1; Schlicke, B.; De Cola, L.; Belser, P.; Balzani, V.; Coord. Chem. Rev. 2000, 208, 267.

131. Grosshenny, V.; Harriman, A.; Ziessel, R.; Angew. Chem., Int. Ed. 1996, 34, 2705; Hush, N. S.; Reimers, J. R.; Hall, L. E.; Johnston, L. A.; Crossley, M. J.; Ann. N.Y. Acad. Sci. 1998, 852, 1; Ratner, M. A.; Davis, B.; Kemp, M.; Mujica, V.; Roitberg, A.; Yaliraki, S.; Ann. N.Y. Acad. Sci. 1998, 852, 22; Jiang, B. W.; Yang, S. W.; Bailey, S. L.; Hermans, L. G.; Niver, R. A.; Bolcar, M. A.; Jones, W. E.; Coord. Chem. Rev. 1998, 171, 365.

132. Bumm, L. A.; Arnold, J. J.; Cygan, M. T.; Dunbar, T. D.; Burgin, T. P.; Jones, L.; Allara, D. L.; Tour, J. M.; Weiss, P. S.; Science 1996, 271, 1705.

133. Franzen, S.; Goldstein, R. F.; Boxer, S. G.; J. Phys. Chem. 1993, 97, 3040; Reimers, J. R.; Hush, N. S.; Chem. Phys. 1995, 197, 323; Moser, C. C.; Page, C. C.; Farid, R.; Dutton, P. L.; J. Bioenerg. Biomembr. 1995, 27, 263; Collin, J.-P.; Harriman, A.; Heitz, V.; Odobel, F.; Sauvage, J.-P.; Coord. Chem. Rev. 1996, 148, 63. 
134. Schuhmann, W.; Biosens. Bioelectron. 1995, 10, 181; Cygan, M. T.; Dunbar, T. D.; Arnold, J. J.; Bumm, L. A.; Shedlock, N. F.; Burgin, T. P.; Jones, L.; Allara, D. L.; Tour, J. M.; Weiss, P. S.; J. Am. Chem. Soc. 1998, 120, 2721; Yaliraki, S. N.; Kemp, M.; Ratner, M. A.; J. Am. Chem. Soc. 1999, 121, 3428 .

135. Finklea, H. O.; Electroanal. Chem. 1996, 19, 109.

136. Hsu, C. P.; Marcus, R. A.; J. Chem. Phys. 1997, 106, 584; Weiss, P. S.; Bumm, L. A.; Dunbar, T. D.; Burgin, T. P.; Tour, J. M.; Allara, D. L.; Ann. N.Y. Acad. Sci. 1998, 852, 145; Bumm, L. A.; Arnold, J. J.; Dunbar, T. D.; Allara, D. L.; Weiss, P. S.; J. Phys. Chem. B 1999, 103, 8122; Willner, I.; Katz, E.; Angew. Chem., Int. Ed. 2000, 39, 1181; Dunbar, T. D.; Cygan, M. T.; Bumm, L. A.; McCarty, G. S.; Burgin, T. P.; Reinerth, W. A.; Jones, L.; Jackiw, J. J.; Tour, J. M.; Weiss, P. S.; Allara, D. L.; J. Phys. Chem. B 2000, 104, 4880.

137. Tour, J. M.; Reinerth, W. A.; Jones, L.; Burgin, T. P.; Zhou, C. W.; Muller, C. J.; Deshpande, M. R.; Reed, M. A.; Ann. N.Y. Acad. Sci. 1998, 852, 197; Martin, R. E.; Diederich, F.; Angew. Chem., Int. Ed. Engl. 1999, 38, 1350.

138. Kochi, J. K.; Angew. Chem., Int. Ed. 1988, 27, 1227; Kochi, J. K.; Bockman, T. M.; Adv. Organomet. Chem. 1991, 33, 51; Kochi, J. K.; Pure Appl. Chem. 1991, 63, 255; Kochi, J. K.; Acc. Chem. Res. 1992, 25, 39; Kochi, J. K.; Adv. Phys. Org. Chem. 1994, 29, 185.

139. Yagi, M.; Nagai, K.; Onikubo, T.; Kaneko, M.; J. Electroanal. Chem. 1995, 383, 61; Zhang, J.; Yagi, M.; Hou, X. H.; Kaneko, M.; J. Electroanal. Chem. 1996, 412, 159; Yagi, M.; Kinoshita, K.; Nagoshi, K.; Kaneko, M.; Electrochim. Acta 1998, 43, 3277; Yagi, M.; Kinoshita, K.; Kaneko, M.; Electrochim. Acta 1999, 44, 2245; Yagi, M.; Fukiya, H.; Kaneko, T.; Aoki, T.; Oikawa, E.; Kaneko, M.; J. Electroanal. Chem. 2000, 481, 69.

140. Purcell, S. T.; Garcia, N.; Binh, V. T.; Jones, L.; Tour, J. M.; J. Am. Chem. Soc. 1994, 116, 11985; Doescher, M. S.; Tour, J. M.; Rawlett, A. M.; Myrick, M. L.; J. Phys. Chem. B 2001, 105, 105.
141. Pourbaix, M.; Atlas of Electrochemical Equilibria in Aqueous Solution, $2^{a}$ ed., Permagon Press: Oxford, 1974; Birge, R. R.; Annu. Rev. Phys. Chem. 1990, 41, 683; Babcock, G. T.; Wikström, M.; Nature 1992, 356, 301; Malmström, B. G.; Acc. Chem. Res. 1993, 26, 332; Tommos, C.; Babcock, G. T.; Acc. Chem. Res. 1998, 31, 18; Cukier, R. I.; Nocera, D. G.; Annu. Rev. Phys. Chem. 1998, 49, 337; Spencer, S. J.; Blaho, J. K.; Lehnes, J.; Goldsby, K. A.; Coord. Chem. Rev. 1998, 174, 391.

142. Rocha, R. C.; Rein, F. N.; Toma, H. E.; J. Braz. Chem. Soc. 2001, 12, 234

143. Reimers, J. R.; Lu, T. X.; Crossley, M. J.; Hush, N. S.; Chem. Phys. Lett. 1996, 256, 353; Tour, J. M.; Kozaki, M.; Seminario, J. M.; J. Am. Chem. Soc. 1998, 120, 8486 .

144. Barigelletti, F.; Flamigni, L.; Balzani, V.; Collin, J.-P.; Sauvage, J.-P.; Sour, A.; Constable, E. C.; Thompson, A. M. W. C.; J. Am. Chem. Soc. 1994 116, 7692; Wagner, R. W.; Lindsey, J. S.; J. Am. Chem. Soc. 1994, 116, 9759; Kalyanasundaram, K.; Gratzel, M.; Coord. Chem. Rev. 1998, 177, 347; Belser, P.; Bernhard, S.; Blum, C.; Beyeler, A.; De Cola, L.; Balzani, V.; Coord. Chem. Rev. 1999, 192, 155.

145. Bissell, R. A.; De Silva, A. P.; Gunaratne, H. Q. N.; Lynch, P. L. M.; Maguire, G. E. M.; Sandanayake, K. R. A. S.; Chem. Soc. Rev. 1992, 187; De Cola, L.; Balzani, V.; Barigelletti, F.; Flamigni, L.; Belser, P.; von Zelewski, A.; Frank, M.; Vögtle, F.; Inorg. Chem. 1993, 32, 5228; Goulle, V.; Harriman, A.; Lehn, J.-M.; J. Chem. Soc., Chem. Commun. 1993, 1034; Harriman, A.; Ziessel, R.; Coord. Chem. Rev. 1998, 171, 331.

146. Murrell, J. N.; J. Am. Chem. Soc. 1959, 81, 5037; Murrell, J. N.; Quart. Rev. (London) 1961, 15, 191.

147. Lever, A. B. P.; Inorganic Electronic Spectroscopy, 2a ed., Elsevier: Amsterdam, 1985

148. Creutz, C.; Newton, M. D.; Sutin, N.; J. Photochem. Photobiol., A 1994 $82,47$.

149. A expressão aproximada final de $f_{\text {osc }}$ assume que o cálculo será efetuado para uma banda simétrica com um perfil tipicamente Gaussiano.

150. Isso é rigorosamente verdadeiro somente para bandas com formato Gaussiano $^{5,60}$ 